ARTICLE

DOI: $10.1038 / \mathrm{s} 41467-018-05582-\mathrm{x}$

\title{
NOVA1 regulates hTERT splicing and cell growth in non-small cell lung cancer
}

Andrew T. Ludlow (1) 1,2, Mandy Sze Wong1,3, Jerome D. Robin ${ }^{1,4}$, Kimberly Batten ${ }^{1}$, Laura Yuan ${ }^{1}$, Tsung-Po Lai ${ }^{1}$, Nicole Dahlson ${ }^{1}$, Lu Zhang${ }^{1}$, Ilgen Mender ${ }^{1}$, Enzo Tedone ${ }^{1}$, Mohammed E. Sayed ${ }^{1,2}$, Woodring E. Wright (10 ${ }^{1} \&$ Jerry W. Shay ${ }^{1}$

\begin{abstract}
Alternative splicing is dysregulated in cancer and the reactivation of telomerase involves the splicing of TERT transcripts to produce full-length (FL) TERT. Knowledge about the splicing factors that enhance or silence FL $h T E R T$ is lacking. We identified splicing factors that reduced telomerase activity and shortened telomeres using a siRNA minigene reporter screen and a lung cancer cell bioinformatics approach. A lead candidate, NOVA1, when knocked down resulted in a shift in $h T E R T$ splicing to non-catalytic isoforms, reduced telomerase activity, and progressive telomere shortening. NOVA1 knockdown also significantly altered cancer cell growth in vitro and in xenografts. Genome engineering experiments reveal that NOVA1 promotes the inclusion of exons in the reverse transcriptase domain of $h T E R T$ resulting in the production of FL hTERT transcripts. Utilizing hTERT splicing as a model splicing event in cancer may provide new insights into potentially targetable dysregulated splicing factors in cancer.
\end{abstract}

\footnotetext{
${ }^{1}$ Department of Cell Biology, UT Southwestern Medical Center, 5323 Harry Hines Boulevard, Dallas, TX 75390, USA. ${ }^{2}$ School of Kinesiology, University of Michigan, 401 Washtenaw Ave., Ann Arbor, MI 48109, USA. ${ }^{3}$ Present address: Cold Spring Harbor Laboratories, One Bungtown Road, Cold Spring Harbor, New York, NY 11724, USA. ${ }^{4}$ Present address: Aix-Marseille University, Marseille Medical Genetics (MMG), UMR125, Marseille 13385, France.

Correspondence and requests for materials should be addressed to A.T.L. (email: atludlow@umich.edu)
} 
T elomerase is a tightly regulated ribonucleoprotein complex (RNP) that maintains or lengthens human telomeres by adding 5'-TTAGGG repeats. Telomerase consists minimally of a reverse transcriptase (RT) protein catalytic subunit (hTERT) and a template RNA, telomerase RNA $\left(h T E R C^{1}\right)$. Embryonic and transit amplifying stem cells have telomerase activity while most somatic cells do not. However, $85-90 \%$ of cancers utilize telomerase activity to maintain telomeres ${ }^{2}$. In most human cancers, the limiting factor in telomerase activity is the expression level of the RT, hTERT. hTERT is regulated by transcriptional and post-transcriptional mechanisms ${ }^{3-5}$. Transcriptional regulation of $h T E R T$ has been extensively studied, however the findings do not completely explain how telomerase is regulated in cancer. How the transcribed messenger RNA is processed (i.e., RNA-processing events), which is critical for determining if active telomerase is produced or not, is less well understood. One such RNA-processing regulatory mechanism is alternative splicing, which contributes to protein diversity and transcript abundance ${ }^{6} . h T E R T$ produces a transcript containing 16 exons that can be spliced into multiple isoforms ${ }^{7-10}$, including the fulllength (FL) RT competent form. In tumor cells and dividing stem cells, FL $h T E R T$ and several spliced variants are co-expressed at detectable levels. Since telomerase activity is almost universally activated in human cancer, further research into the mechanisms that regulate $h T E R T$ mRNA processing, specifically alternative splicing, may provide additional clues about telomerase regulation in cancer and importantly could elucidate new candidate genes to target for telomerase inhibition and for anticancer therapies.

Of the $h T E R T$ splice isoforms, the four major isoforms that have been studied involve exons 5-9, which encode the RT domain of hTERT (Supplementary Table 1, Supplementary Figure 6). The four major isoforms are a result of splicing of regions termed "alpha" and "beta" located within exons $5-9^{10}$. Only the "FL" version $(\alpha+\beta+; \mathrm{FL})$ containing all five intact exons of the RT domain has the potential to encode catalytically active enzyme $^{8,10,11}$. The other isoforms are generated by skipping of exons 7 and $8(\alpha+\beta-;$ minus beta), which introduces a frameshift and premature stop codon in exon 10, the skipping of 36 nucleotides (nts) of exon 6 ( $\alpha-\beta+$; minus alpha), which is in frame and generates a dominant-negative RT incompetent telomerase $^{10}$, and $(\alpha-\beta-;$ minus alpha-beta), which has both skipping events $\left({ }^{10}\right.$; Supplementary Table 1$)$. Other variants of $h T E R T$ exist that result from splicing events outside of the RT domain ${ }^{12}$ (Supplementary Table 1). The important regulatory sequences and splicing factors that bind $h T E R T$ pre-mRNAs to produce the RT competent versus RT-deficient $h T E R T$ splice isoforms are not well described.

Very few investigations into the cis- and trans-acting factors that regulate splicing of $h T E R T$ have been performed. Our group previously identified highly conserved sequences in old world primates, including humans, that regulate hTERT splicing choice $^{13,14}$. However, little is currently known about the transacting factors that bind these regulatory regions. Thus, identification of such proteins would close a significant gap in telomerase regulation knowledge and also potentially identify protein targets to shift the splicing of $h T E R T$ message to inactive forms to reduce telomerase activity, progressively shorten telomeres, and ultimately leading to reduced tumor growth in vivo. RNA-binding proteins target multiple genes; thus, it is likely that identification of a protein that targets $h T E R T$ may impact other important pathways that are cancer cell dependencies.

Recently, three splicing proteins, SRSF11, hnRNPL, and $h n R N P H 2$, when overexpressed in cancer cells were identified to potentially regulate $h T E R T$ minus beta splicing choice using an hTERT minigene ${ }^{15}$. There are more than 500 RNA-binding proteins encoded in the genome and splicing is the result of cellular context, RNA secondary structure, RNA editing, and competition for splice sites, therefore much is left to be learned concerning $h T E R T$ splicing regulation ${ }^{16-18}$.

To address the protein networks that regulate the alternative splicing of FL $h T E R T$ in cancer cells, we took two approaches: a hTERT dual-luciferase minigene splicing reporter RNAi screen and a bioinformatic analysis of a panel of highly characterized human lung cancer cell lines to identify genes that may regulate hTERT splicing and other cancer cell phenotypes. These two approaches identified neuro-oncological ventral antigen 1 (NOVA1) as a candidate gene. In non-small cell lung cancer cells that express high levels of NOVA1, we found that stable reduction in NOVA1 levels shifted $h T E R T$ splicing toward inactive transcripts, reduced telomerase activity, which led to progressively shortened telomeres. We also demonstrated that NOVA1 knockdown reduced migration through extracellular matrices, and resulted in smaller tumors in vivo. Thus, the experiments described in the present report provide a mechanistic view of how cancer cells regulate $h T E R T$ splicing.

\section{Results}

hTERT minigene small interfering RNA screen of RNAbinding proteins. To investigate the protein factors involved in alternative splicing of $h T E R T$ we performed a small interfering RNA (siRNA) screen in HeLa cells stably expressing an $h T E R T$ minigene splicing reporter (Fig. 1). The minigene used in this study allowed for luciferase-based measurements of hTERT "FL" (intact exons 6-8) and minus beta hTERT (skipping of exons 7 and 8). Fluc indicates minus beta splicing (inactive hTERT) and FL hTERT is indicated by Rluc (Fig. 1a).

We obtained pools of four siRNAs to 516 RNA-binding protein genes. HeLa cells containing the minigene reporter were transfected with the siRNAs and luciferase measurements were made $72 \mathrm{~h}$ later (Fig. 1a). The screen repeat runs were highly correlated $\left(R^{2}=0.794\right.$, Pearson's $r$, Supplementary Figure 1C). Of the 528 siRNAs tested, 97 siRNAs targeting 93 unique genes resulted in a twofold or greater increase in minus beta splicing and 20 siRNAs targeting 17 genes resulted in a twofold or greater increase in FL splicing (a list of these gene identifiers can be found in Supplementary Data file 1). Our objective was to find targets that had cancer specificity (i.e., not widely or highly expressed across normal adult human tissues) for potential follow-up studies; thus, we focused our follow-up studies on the 93 genes that when knocked down resulted in twofold or greater increases in the ratio of minus beta to FL. We pursued a parallel bioinformatics approach to narrow down the list of candidate genes using a highly characterized panel of human non-small cell lung cancer cell lines.

Bioinformatics analysis of 516 RNA-binding proteins. We measured telomere biology phenotypes (telomerase activity, hTERT expression, and telomere length ${ }^{19}$ ) in 17 wellcharacterized lung cancer cell lines and correlated expression of splicing factors and telomere biology between these cell lines to narrow down our target gene list (Supplementary Figure 1D, Supplementary Figure 1E). We found that telomere length and telomerase activity were significantly correlated $\left(R^{2}=0.51, p=\right.$ 0.001 , Pearson's $r$, Supplementary Figure 1G). hTERT mRNA expression of exons $7 / 8$ and exons $15 / 16$ showed a modest but significant correlation with telomere length $\left(R^{2}=0.38, p=0.008\right.$ for exons $7 / 8$ and $R^{2}=0.30, p=0.02$ for exons $15 / 16$, Pearson's $r$; Supplementary Figure $1 \mathrm{H})$. Telomerase activity correlated with exons 7/8 expression of $h$ TERT $\left(R^{2}=0.27, p=0.03\right.$, Pearson's $r$; Supplementary Figure 1I). We quantified $h T E R T$ splice isoforms 
a
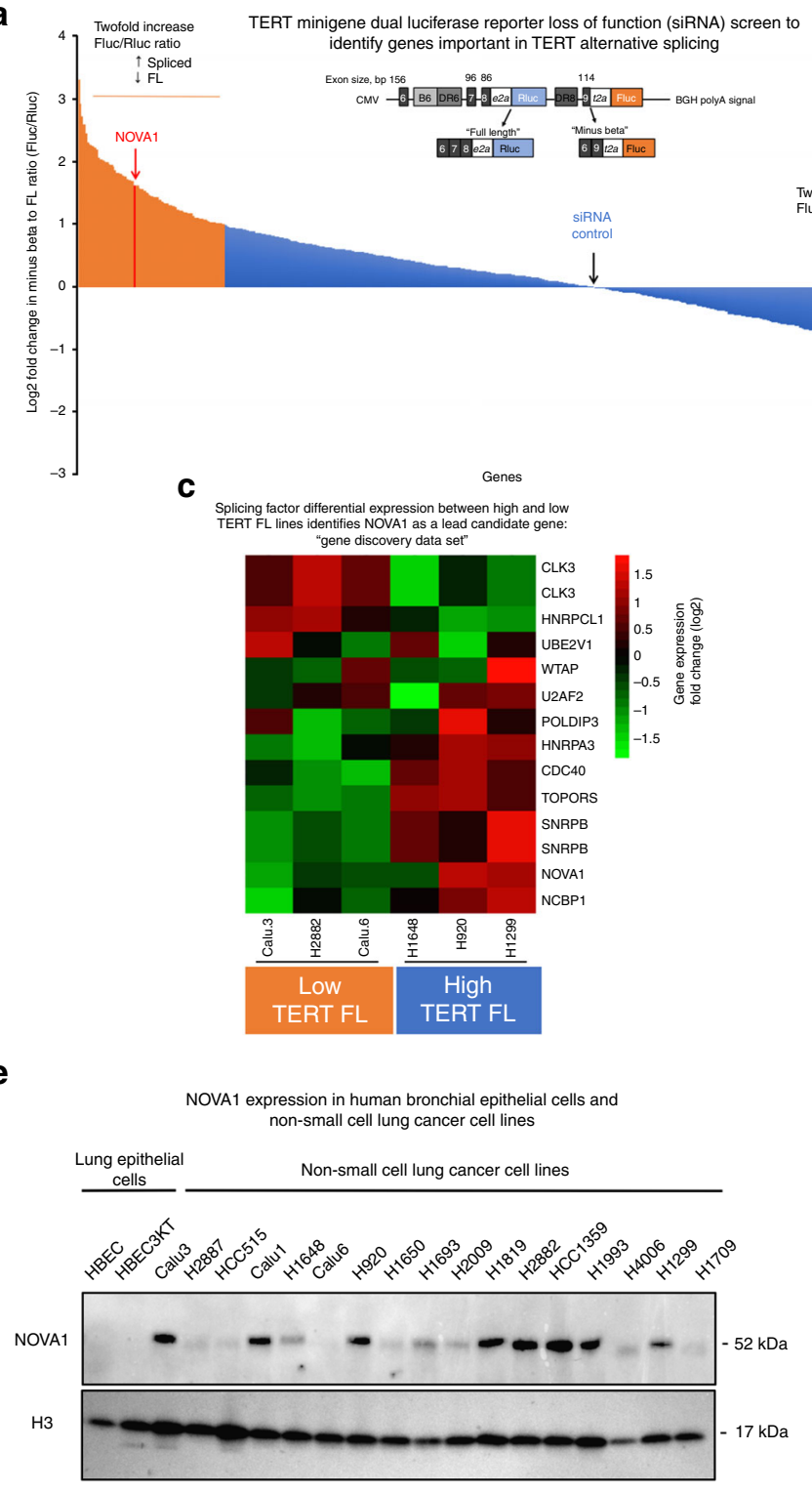

b

hTERT splicing profiles of 17 non-small cell lung cancer cell lines

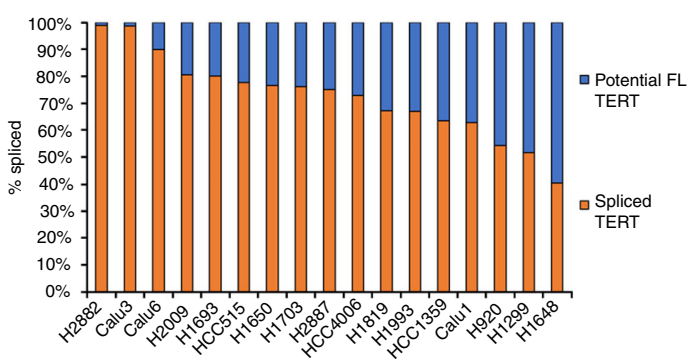

d 'Network discovery' - differential expression analysis of splicing
factors in cell lines reveals a splicing network related to TERT FL

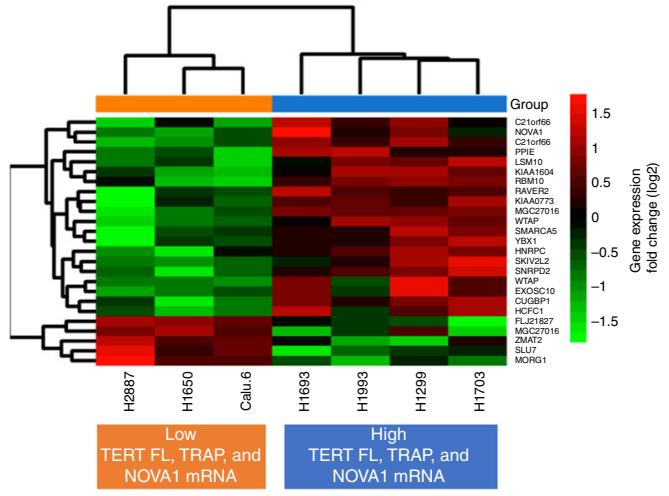

f

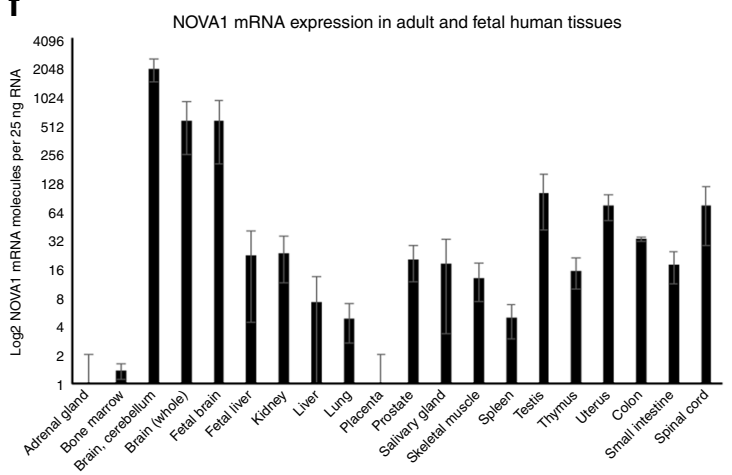

\section{g}

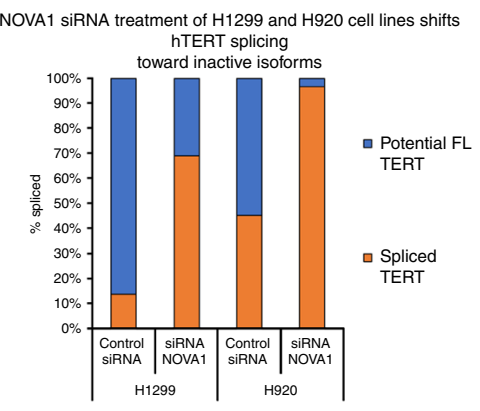

h

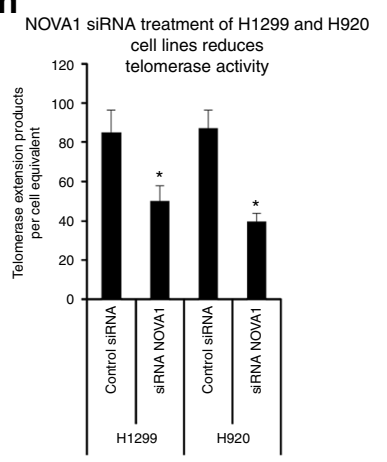

(Fig. 1b, primer sequences are in Supplementary Table 2), we choose to analyze cell lines that differed significantly by the percentage of FL $h T E R T$ expression, percentage of minus beta $h T E R T$ expression, and telomerase activity (three lines at each extreme, Fig. 1c, "Gene Discovery Data set"). We correlated the expression data of 516 RNA-binding proteins across six cell lines (differential expression analysis ( $t$ tests), false-discovery rate corrected with Benjamini-Hochberg procedure) to the amount of potential FL $h T E R T$ as measured by our splice isoform assays. This analysis revealed 12 genes whose expression significantly correlated to the amount of FL hTERT (heatmap shows differential expression of the 12 genes that correlated to hTERT 
Fig. 1 hTERT alternative splicing is regulated by a network of RNA-binding proteins. a TERT minigene reporter construct and products. CMV: human cytomegalovirus immediate-early enhancer and promoter, Bp: base pair, B6: variable nucleotide repeat in intron 6 from ref. ${ }^{13}$, DR6 and DR8: direct repeat 6 or 8, respectively, from ref. ${ }^{13}$, BGH polyA: signal bovine growth hormone polyadenylation signal. $\mathbf{b} h T E R T$ steady-state isoform/splicing profile in non-small cell lung cancer cell lines $(n=3)$. c Differential expression analysis of splicing factors that correlated with high $h T E R T$ full-length expression in six non-small cell lung cancer cell lines. Log2 fold change in gene expression between the cell lines. CLK3 and SNRPB appear twice in the heatmap because two different microarray probes were differentially expressed between the high $h T E R T$ and low $h T E R T$ lines. $\mathbf{d}$ Differential expression analysis of splicing factors related to hTERT full-length expression, telomerase activity, and NOVA1 mRNA expression in the seven non-small cell lung cancer cell lines. Log2 fold change in gene expression between the cell lines. e Expression of NOVA1 protein and histone H3 protein in normal (HBECs) and cancerous lung cell lines. Representative western blot images are shown $(n=3)$. f Expression of NOVA1 mRNA by RT-ddPCR in a panel of human fetal and adult tissues. RT reverse transcription, ddPCR droplet digital PCR $(n=3)$. g siRNA knockdown of NOVA1 in H1299 and H920 lung cancer lines shifts hTERT splice isoform proportions as determined using RT-ddPCR $(n=6)$. h siRNA knockdown of NOVA1 in H1299 and H920 lung cancer lines reduces telomerase activity per cell equivalent (50 cell equivalents, $n=6$, Student's $t$ test set at ${ }^{*} p<0.05$ for significance). Data are expressed as means and standard error of the mean where applicable. Supplementary data associated with this figure can be found in Supplementary Figure 1

expression in 6 non-small cell lung cancer cell lines; Fig. 1c). Of these 12 genes, 4 genes overlapped with the 93 genes that also changed (twofold increase in reporter minus beta $h T E R T$ signal compared to control) in the minigene screen (SNRPB, NOVA1, $U 2 A F 2$, and $C D C 40$ ). NOVA1 was the top-ranking tissue-specific RNA-binding protein associated with cancer ${ }^{20,21}$, and in silico analysis revealed binding sites for NOVA1 within the hTERT locus. Of the 37 proteins that had a greater impact on switching hTERT splicing in the minigene assay, NOVAl was the only factor with an expression profile that was not ubiquitous indicating the potential for therapeutic targeting. Thus, NOVA1 was chosen for further analysis.

We hypothesized that NOVA1 may mark a network of splicing factors related to high levels of $h T E R T$ FL mRNA ${ }^{22}$. Hierarchical clustering analysis (divisive) was used to separate and prioritize cell lines based on telomerase biology ( $\log 2$-transformed telomerase and percentage of FL hTERT) and expression of NOVA1 mRNA (log2-transformed as measured by droplet digital PCR (ddPCR)). Using two groups of cell lines (seven cell lines total: four with high telomerase, percentage of FL hTERT, NOVA1 compared to three low telomerase, percentage of FL hTERT, and NOVA1) we compared the expression profiles of the 516 RNA-binding proteins and splicing factors (dendrogam of hierarchical clustering analysis of cell lines Supplementary Figure 1I). This expression analysis revealed 22 genes with different expression patterns between the two groups of lung cancer cell lines (Fig. 1d; heatmap for "Network Discovery Data set"). Next, we probed the overlapping genes between this gene list (22) and the minigene list (93) and observed that 7 genes overlapped (SNRPD2, CWC22, NOVA1, RBM10, ZMAT2, SLU7, and FAM131B). Ten genes came out in at least two of the three screens and caused a twofold or greater change in the minigene reporter screen (Supplementary Figure 1J; Supplementary Data file 1 shows tabulated results of all three screens, siRNA sequences, and the overlap analyses).

NOVA1 protein was not expressed in normal human bronchial epithelial cells (HBECs), and was overexpressed in $71 \%$ of our lung cancer cell lines (12 of 17 lines; Fig. 1e, Supplementary Figure $1 \mathrm{~F})$. We correlated the expression of NOVA1 mRNA and protein to the percentage of hTERT FL mRNA and observed a significant correlation in both cases (Pearson's $r, R^{2}=0.25, p=$ 0.039 for mRNA; $R^{2}=0.34, p=0.01$ for protein; Supplementary Figure $1 \mathrm{~L}$ and $\mathrm{M}$, respectively). NOVA1 mRNA expression and NOVA1 protein expression were significantly correlated in our non-small cell lung cancer cell lines $\left(R^{2}=0.50, p=0.001\right.$, Pearson's $r$; Supplementary Figure $1 \mathrm{~N}$ ). We also measured the expression of NOVA1 mRNA in a panel of human adult and fetal tissues, observing low expression in tissues with the exception of brain (as expected), reproductive (testis), and fetal organs (Fig. 1f).
To confirm the results of the minigene screen and the bioinformatics correlation analysis, we performed short-term siRNA knockdown experiments (Fig. 1g, h). hTERT potential FL mRNA levels were reduced by $60 \%$ in $\mathrm{H} 1299$ and $50 \%$ in $\mathrm{H} 920$ cells treated with NOVA1 siRNAs compared to cells treated with control non-targeting siRNAs (Fig. 1g). Telomerase enzyme activity was reduced in NOVA1-depleted cells by 2 -fold ( $p=0.05$, Student's $t)$ and 2.5 -fold $(p<0.05$, Student's $t)$ in H1299 and H920 cells, respectively compared to control siRNA-treated cells (Fig. $1 \mathrm{~h}^{23}$. Knockdown of NOVA1 was confirmed in both cell lines (Supplementary Figure 1O). Importantly, hTERT steadystate transcripts (exons 15/16) were not significantly decreased by transient knockdown of NOVA1 in either cell line (Supplementary Figure 1P), indicating that NOVA1 knockdown results in a change in splicing and not just a downregulation in transcriptional rate, which is known to affect splicing ${ }^{24}$. We did observe a reduction in exon $7 / 8$ containing transcripts, confirming the reduction in potential FL (Supplementary Figure 1P). These data together with the minigene and bioinformatics analyses support that NOVAl is a key member of a potential network of genes regulating $h T E R T$ alternative splicing.

Long-term depletion of NOVA1 shifts $h$ TERT splicing. Since its initial description, NOVA1 (Ri-antigen) has been observed to be overexpressed in neuro-endocrine cancers such as breast, lung, and brain cancers ${ }^{20,21,25-27}$. NOVA1 contains three K-homology domains and binds RNA at YCAY $(\mathrm{Y}=\mathrm{C}$ or $\mathrm{T})$ clusters commonly found though out the genome (1 in $64 \mathrm{nts})^{28,29}$. NOVA1 YCAY RNA-binding motifs are found in hTERT introns and exons. We knocked down NOVA1 in three different cell lines: two that express NOVA1 (H1299 and H920) and one that does not express NOVA1 (Calu6, as a control for off-target effects of the short hairpin RNA (shRNA) sequence). Stable knockdown of NOVA1 reduced telomere length and telomerase activity in the two cell lines that express NOVA1 (Fig. 2a, H1299 cells, Supplementary Figure 2A). NOVA1 protein and mRNA levels were reduced by about 50\% (Fig. 2c, Supplementary Figure 2D). NOVA1 knockdown reduced the proportion of FL $h T E R T$ message (Fig. 2b; Supplementary Figure 2B) and decreased telomerase activity about $50 \%$ ( $p=0.05$, Student's $t$, Fig. 2d; Supplementary Figure $2 \mathrm{C}$ ), which was sufficient to reduce telomere length in both H1299 and H920 cells. The >50\% telomerase activity reduction observed leading to progressive telomere shortening is consistent with human diseases of $h T E R T$ haploinsufficiency that also result in clinically significant shortened telomeres. The long-term depletion of NOVA1 also reduced the steady-state transcript levels of hTERT (Fig. 2d, Supplementary Figure 2E). To demonstrate that the shRNA was on target for NOVA1, we transduced H1299 cells with a retroviral 6X MYC-tagged cDNA construct coding for FL NOVA1 with the shRNA seed sequence 
a

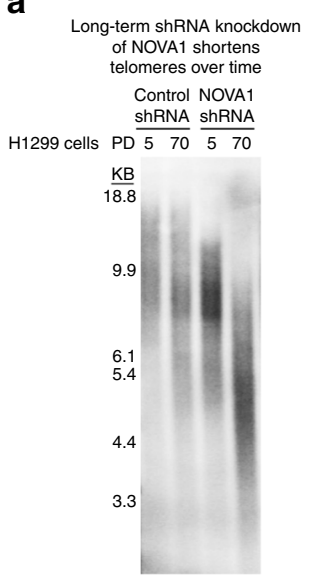

b

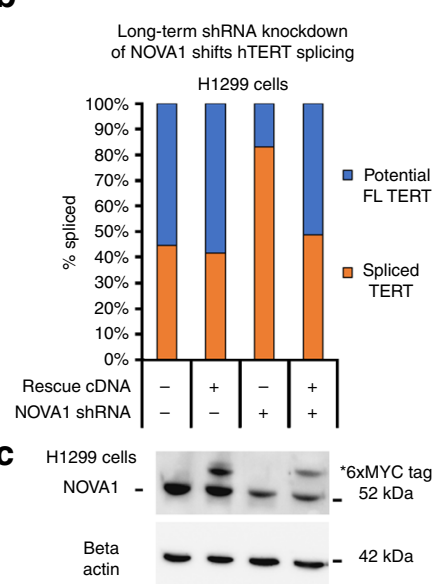

d

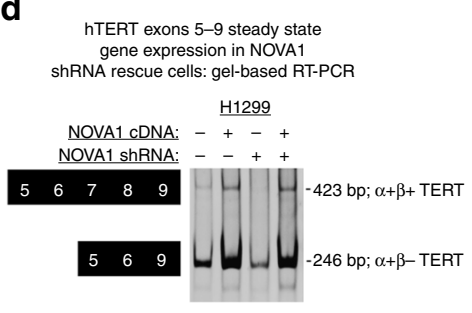

e

Long-term shRNA knockdown of NOVA1 reduces

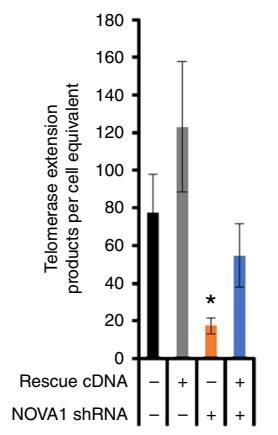

f

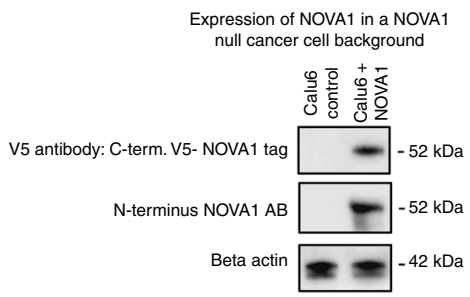

g

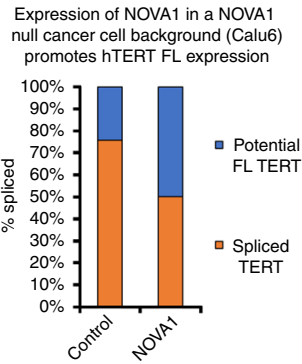

h

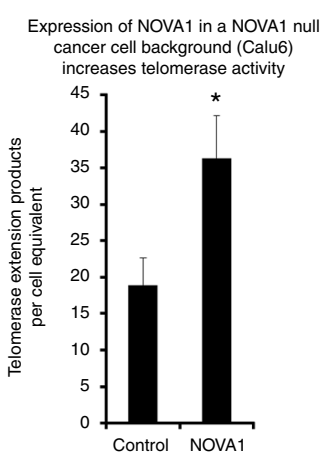

Fig. 2 Long-term reduction of NOVA1 progressively shortens telomeres. a Terminal restriction fragment length (TRF-Southern blot) analysis of control shRNA or NOVA1 shRNA at two population doublings (PD). b Rescue of shRNA knockdown of NOVA1 with a shRNA mutant cDNA in H1299 cells (stable cell lines were measured a minimum of six times over several passages). hTERT splicing was determined with RT-ddPCR assays. c Western blot of NOVA1 shRNA rescue in $\mathrm{H} 1299$ cells (representative image of stable cell lines, measured three times over three passages in culture). $\mathbf{d} h T E R T$ expression in rescue H1299 cells as determined by RT-PCR of exons 5-9 (representative image; $n=3$ ). e Rescue of shRNA knockdown of NOVA1 with a shRNA mutant cDNA partially restores telomerase activity in $\mathrm{H} 1299$ cells $(n=6)$. Telomerase activity was determined with droplet digital TRAP (ddTRAP with 50 cell equivalents added to the assay). Student's $t$ test set at ${ }^{*} p<0.05$ for significance. $\mathbf{f}$ Western blot of V5-tagged NOVA1 expression in Calu6 cells (representative image of stable cell lines). $\mathbf{g}$ hTERT splicing profile in Calu6 cells with and without NOVA1 $(n=6)$. hTERT splicing was determined with RTddPCR assays. $\mathbf{h}$ Telomerase enzyme activity (ddTRAP with 50 cell equivalents added to the assay) in Calu6 cells with and without NOVA1 ( $n=3$ ). Student's $t$ test set at ${ }^{\star} p<0.05$ for significance). Data are expressed as means and standard error of the mean where applicable. ${ }^{\star} p<0.05$. RT: reverse transcription, ddPCR: droplet digital PCR. + indicates presence of shRNA or cDNA construct. - indicates absence of shRNA or cDNA construct (b, d, e). Supplementary data associated with this figure can be found in Supplementary Figure 2

mutated. The shRNA-resistant NOVA1 cDNA was able to rescue hTERT splicing changes, transcript levels, and telomerase enzyme activity (Fig. $2 \mathrm{~b}, \mathrm{~d}, \mathrm{e}$ ). These experiments show that NOVA1 is mechanistically linked to $h T E R T$ and that the observed phenotypes associated with knockdown are on target. Further, targeting NOVA1 resulted in a potent effect on $h T E R T$, not only by shifting the splicing away from FL $h T E R T$ transcripts but also by reducing overall $h T E R T$ transcription, which is a different observation compared to the transient siRNAs. The reduced overall $h T E R T$ mRNA levels are important to consider with recent data indicating that $h T E R T$ may have non-canonical roles ${ }^{30,31}$. To explain how the NOVA1 rescue returned $h T E R T$ transcript levels to control values, one possibility is that the increased levels of hTERT (Fig. 2d) is due to a transcription factor being spliced or another upstream event that NOVA1 is involved in that results in increased expression of $h T E R T$. Recent RNA-sequencing data indicate that NOVA1 may regulate the expression of transcription factors that could be acting upstream of $h T E R T$ in cancer cells $\mathrm{s}^{32}$.

To determine the effects on cell growth of knocking NOVA1 down in non-transformed lung cells, we introduced control (non- silencing) and NOVA1 shRNAs into HBECs and observed no significant growth defect (Supplementary Figure 2M). To further determine the effects of NOVA1 reduction on normal cell growth, we used a siRNA treatment strategy to significantly reduce NOVA1 expression in a normal diploid fibroblast cell strain (BJ fibroblasts). Over the course of the experiment there was no inhibition in growth or viability of the BJ fibroblasts treated with NOVA1 siRNAs compared to control-treated siRNAs (Supplementary Figure $2 \mathrm{O}$ and $\mathrm{P}$ ). The siRNA-induced knockdown of NOVA1 at both the protein (Supplementary Figure 2Q) and mRNA level was significantly reduced compared to controltreated cells (Supplementary Figure 2R).

We attempted to knockout NOVA1 with CRISPR/Cas9 genome-editing methods in H1299 lung cancer cells but were unable to obtain survival clones, suggesting that NOVA1 may be critical in cell survival pathways in H1299 lung cancer cells. When we knocked down NOVA1 with shRNAs in H2882, another non-small cell lung cancer cell line, the cells only divided twice in 90 days following selection (Supplementary Figure 2S). H2882 cells have very robust levels of NOVA1 (Fig. 1e), adding strength to the idea that NOVA1 may be a critical gene to cancer 
a
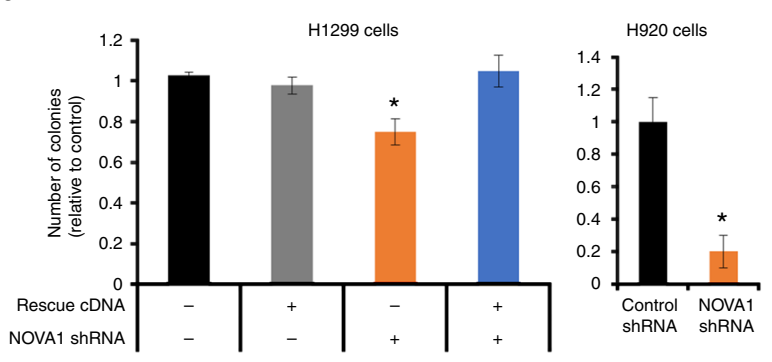

C

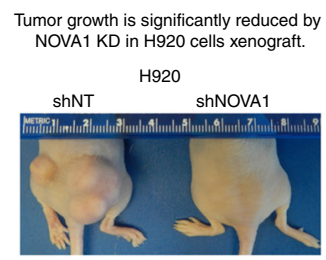

e

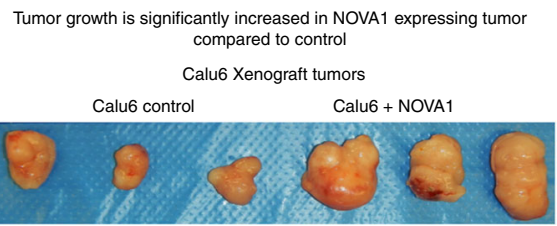

b Invasion through matrigel is reduced by NOVA1 KD in H1299 and H920 cells.
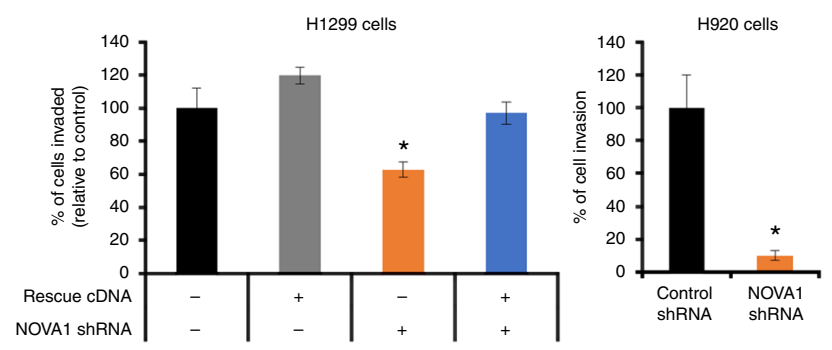

d
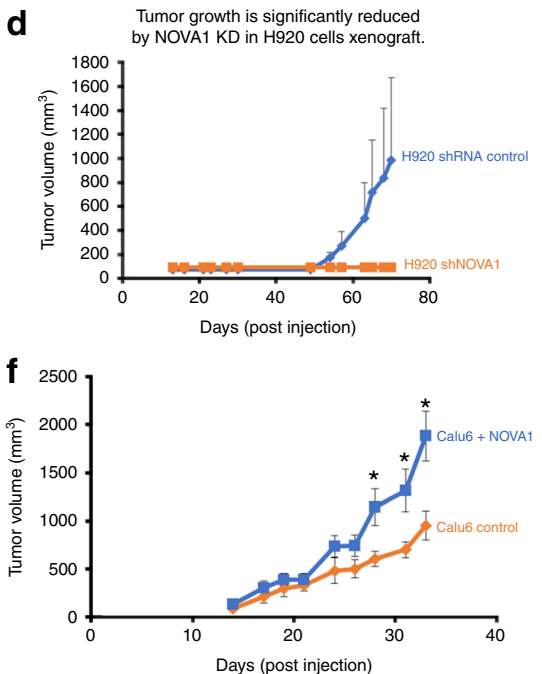

Fig. 3 Manipulation of NOVA1 in cancer cells alters xenograft tumor growth. a Knockdown of NOVA1 in H1299 and H920 cells reduced anchorageindependent cell growth compared to controls (soft agar colony formation assays, $n=3$ for each condition and cell line). + indicates presence of shRNA or cDNA construct. - indicates absence of shRNA or cDNA construct. b Knockdown of NOVA1 in H1299 and H920 cells reduced migration through an extracellular matrix (Boyden Chamber assay) compared to controls ( $n=3$ for each condition and cell line). + indicates presence of shRNA or cDNA construct. - indicates absence of shRNA or cDNA construct $(\mathbf{a}, \mathbf{b})$. c Knockdown of NOVA1 in H920 cells significantly reduced xenograft growth compared to controls. Left panel shows representative images of the hind quarters of nude mice. $\mathbf{d}$ Growth curves of xenograft tumors during the experiment $(n=4$ injections for each condition, 2 mice per condition). e Expression of NOVA1 in Calu6 cells results in larger xenograft tumors compared to Calu6 controls (a non-small cell lung cancer cell line lacking NOVA1 expression). Left panel shows representative images of tumors from Calu6 and Calu6 plus NOVA1. Same stable cell line as used in Fig. 2 and Supplementary Figure 2. f Right panel shows growth curves of xenograft tumors during the experiment ( $n=6$ injections for each condition, 3 mice per condition). Data are expressed as means and standard error of the mean where applicable. All tests shown are Student's $t$ test set at ${ }^{\star} p<0.05$ for significance. KD: knockdown. Note-an error bar on the controls of a-c was calculated by first generating the mean of the controls and expressing each control relative to this value. We then calculated a standard deviation and standard error based on the variance observed between these normalized values. Supplementary data associated with this figure can be found in Supplementary Figure 3

cell survival. We observed that a different shRNA targeting NOVA1 in H1299 cells significantly slowed growth and/or was rapidly silenced (Supplementary Figures $2 \mathrm{~T}$ and $\mathrm{U}$ ).

Expression of NOVA1 in cancer cells shifts $h T E R T$ splicing. To determine if expression of NOVA1 in a NOVA1-negative cancer cell line promotes $h T E R T$ FL splicing and increases in telomerase activity, we transduced Calu6 cells with a lentiviral vector containing a V5-C terminus-tagged NOVA1 FL cDNA. Overexpression of NOVA1 with both tagged (V5 epitope antibody) and $\mathrm{N}$ terminus NOVA1 antibodies (Fig. 2f) was confirmed. Telomerase activity increased twofold $(p=0.05$, Student's $t$, Fig. $2 \mathrm{~h}$ ) as did the proportion of hTERT FL transcripts (23\% vs. $50 \%$; Fig. $2 \mathrm{~g}$ ) in NOVA1-expressing cells compared to control empty vector cells. In these experiments, telomere length was slightly elongated by overexpression of NOVA1 (Supplementary Figure $2 \mathrm{~N}$ ) in line with the NOVA1 knockdown data.

NOVA1 knockdown reduces cancer cell growth phenotypes. We assayed the tumorigenic properties of NOVA1 knockdown cells. NOVA1 has a known role in breast and lung cancers ${ }^{33}$ and is important during normal development for growth, survival, migration, and apoptosis $32,34,35$. NOVA1 knockdown H1299 cells at population 50 formed significantly fewer colonies compared to control and NOVA1 rescue cells $(p<0.05$, Student's $t$, Fig. 3a). NOVA1 knockdown H1299 cells did not invade as efficiently as H1299 shRNA control cells or the NOVA1 rescue H1299 cells using Boyden chamber assays ( $p<0.05$, Student's $t$, Fig. 3b). Finally, in a colony formation assay we observed that NOVA1-depleted H1299 cells formed fewer colonies compared to control shRNA H1299 cells and rescue cells ( $p<0.05$, Student's $t$, Supplementary Figure 3 ). We also assayed cancer growth phenotypes in a second cell line after 50 population doublings, H920, and both anchorageindependent growth and invasive phenotypes were significantly reduced in NOVA1-depleted cells compared to control shRNA cells (Fig. 3a, b; $p<0.05$, Student's $t$ ). Interestingly, the levels or amount of NOVA1 in the cell lines (H1299 moderate NOVA1 compared to high levels of NOVA1 in H920 cells) seems to correlate the response of the cells to knockdown of NOVA1. For instance, the impact of NOVA1 knockdown on colony formation and migration was much greater in H920 cells compared to H1299 cells. In Calu6 
cells, which lack NOVA1 expression, we observed no differences in anchorage-independent growth, invasion, or colony formation between NOVA1 shRNA cells and control shRNA cells, indicating that these effects were due to on-target effects of the shRNA (Supplementary Figure 3).

To directly test if the growth phenotypes observed with NOVA1 knockdown were due to changes in telomere length or telomerase expression, we attempted to rescue the NOVA1 knockdown by expression of an $h T E R T$ cDNA. We introduced an $h T E R T$ cDNA (pMIN-Ub-IRES-Blast lentiviral vector from ref. ${ }^{36}$ ) into H1299 cells that either already had control shRNA or NOVA1 shRNA and were cultured for 50 population doublings. In the H1299 cells with control shRNAs or NOVA1 shRNAs, we confirmed overexpression of hTERT at the mRNA level (Supplementary Figures 3D and E) and increased telomerase activity in the hTERT-transduced cells (Supplementary Figure 3F). Additionally, we introduced NOVA1 and control shRNAs into U2OS cells, which do not depend on telomerase to maintain telomeres but rather use alternative lengthening (ALT) of telomeres to maintain telomeres. Post selection, these cells were expanded and assayed to assess the impact of NOVA1 knockdown on growth independent of telomere length changes. We confirmed NOVA1 knockdown in the U2OS cells (Supplementary Figure 3J). We assayed each of these model systems (manipulated lines derived from H1299 cells and U2OS cells) for clonogenicity and anchorage-independent growth. We observed that NOVA1 knockdown was not bypassed by forced hTERT expression in the H1299 cells with NOVA1 knockdown at population doubling 60 (Supplementary Figure 3H). The H1299 cells with the combination of $h T E R T$ expression and NOVA1 knockdown formed fewer colonies in both assays of growth compared to H1299 cells with control shRNA and hTERT expression (both the NOVA1 knockdown and the NOVA1 knockdown with $h T E R T$ expression formed about $50 \%$ fewer colonies in the colongenicity assay and $40-50 \%$ fewer colonies in the anchorage-independent soft agar growth assay; Supplementary Figure $3 \mathrm{H}$ and I). Interestingly, upon $h T E R T$ overexpression the levels of NOVA1 increased in both the shRNA control and shRNA NOVA1 lines. This observation can indicate at least two possibilities; either a hypermorphic phenotype where TERT is acting in a way it normally would not due to the high expression levels or that a regulatory loop exists between NOVA1 and hTERT. Further, ALT cells with control shRNAs grew substantially better in both growth assays compared to NOVA1 shRNA cells, forming about twice as many colonies in both assays as well (Supplementary Figure $3 \mathrm{~K}$ and $\mathrm{L}$ ). This indicates that beyond the control of telomerase activity and hTERT splicing in cancer cells, NOVA1 is independently involved in pathways related to tumorigenesis and clonogenic growth.

NOVA1 confers a growth and survival advantage of tumor cells. To determine if cell lines with high or low levels of NOVA1 formed tumors in vivo, we injected cells into both hind flanks of immunocompromised mice. We observed three out of four injections of H920 shRNA control cells formed tumors in vivo while only one of four injections of H920 NOVA1 knockdown cells formed tumors (Fig. $3 \mathrm{c}, \mathrm{d}$ ). The tumors derived from the control cell lines were significantly larger compared to the single tumor derived from the NOVA1 knockdown cells. This indicates that NOVA1 knockdown in $\mathrm{H} 920$ cells significantly altered the ability of these lung cancer cells to form tumors in vivo. Further, we injected mice with Calu6 control lentiviral vector cells (a cell line that does not express NOVA1) and compared it to Calu6 cells with ectopic expression of NOVA1. We monitored the tumors for a period of 4 weeks and observed that NOVA1- expressing cells formed bigger tumors compared to the control Calu6 cells (Fig. 3e, f). Similar to the knockdown experiment, the NOVA1 expression conferred a growth advantage in vivo over cells that lacked NOVA1.

NOVA1 interacts with $h T E R T$ pre-mRNA. NOVA1 binds to pre-mRNAs in a sequence-dependent fashion, binding to YCAY $(\mathrm{Y}=\mathrm{C}$ or $\mathrm{U})$ motifs in RNAs ${ }^{37}$. First, we looked in silico at the hTERT locus for YCAY motifs, focusing on sequences (including introns and exons) from exons 5 to 10 (Fig. 4a). It is not possible to use previous public HITS-CLIP databases from mice because of sequence element differences between mice and humans ${ }^{13}$. Since NOVA1 is known to bind to clusters of YCAY motifs we looked for areas of highly concentrated motifs. We found several potential candidate regions in hTERT exons and introns 5 through 10 (Fig. 4a). We performed ultraviolet (UV)-crosslinking and immunoprecipitation (UV-IP) ${ }^{38}$ of H1299 cells and observed an enrichment of the NOVA1:hTERT RNA interaction in hTERT intron 8 (direct repeat 8 , DR8). NOVA1 appeared to bind in a region we previously observed to be involved in the regulation of $h T E R T$ alternative splicing (Fig. $4 \mathrm{a}-\mathrm{c})^{13}$. Our working model is that NOVA1 binds to the DR8 region and promotes splicing of $h T E R T$ to include RT domain-coding exons 7 and 8. This idea is consistent with previous observations suggesting that NOVA1 can act as a splicing enhancer if it binds following an alternatively spliced exon ${ }^{39}$. From in silico analysis we found that DR8 of hTERT, a 258-base pair element, contains 7 YCAY NOVA1binding motifs. Previously, we found that when cells were treated with a 2-O-methyl-antisense oligonucleotide to this region, hTERT minus beta splicing was increased and FL splicing was reduced, supporting the idea that a factor that promotes inclusion of $h$ TERT exons 7 and 8 was blocked ${ }^{13}$. This is consistent with our observations that $h T E R T$ minus beta splicing increases when NOVA1 levels are reduced. To confirm the UV-IP observations, we utilized our $h T E R T$ minigene series that we previously developed, which excludes DR8, the intron 8 region containing the NOVA1-binding motifs, and performed crosslinking and immunoprecipitation followed by RT-ddPCR (CLIP-ddPCR) (Fig. 4d). As additional controls, we included constructs that contained or excluded a highly similar ( $85 \%$ homology) sequence region in intron 6 called direct repeat 6 (DR6; Fig. 4e). Interestingly, DR6, a 254-base pair region in intron 6, contains fewer YCAY (5) motifs than DR8. When DR8 was present we could effectively pull down $h T E R T$ minigene pre-mRNAs, but we could not when DR8 was absent. As a control, we also assayed our CLIP cDNAs for a known NOVA1 target gene, glycine receptor alpha 2 $(G L R A 2)^{40}$. As previously described GLRA2 exon $3 \mathrm{~A}$ and $3 \mathrm{~B}$ are mutually exclusive exons regulated by NOVA1. First, we tested for expression of GLRA2 mutually exclusive exon 3A and 3B usage in HeLa and H1299 cancer cells (Supplementary Figure 4C and $\mathrm{E}$ ), and observed that exon $3 \mathrm{~B}$ of GLRA2 was preferentially used in cancer cells. Next, we looked in our H1299 NOVA1 rescue series to see if GLRA2 was regulated by NOVA1 in cancer cells and indeed found that GLRA2 exon $3 \mathrm{~B}$ is preferentially used when NOVA1 levels are higher (Supplementary Figure 4D) similar to previous studies ${ }^{40}$. Next, we assayed our CLIP cDNAs and observed that GLRA2 was effectively pulled down in all extracts regardless of $h T E R T$ status, indicating that our CLIP was efficient (Supplementary Figure 4E). To further confirm the CLIP and UV-IP observations, we in vitro transcribed a $1 \mathrm{~kb}$ fragment of RNA containing DR8 of $h T E R T$ intron 8 and performed an RNA pull-down assay (Fig. 4f). When we expressed NOVA1 in 293 cells and exposed the lysate to the labeled RNA, we observed effective pull down of NOVA1 (Fig. 4g). These data indicated that NOVA1 was binding to this $1 \mathrm{~kb}$ RNA fragment of $h T E R T$ intron 
a

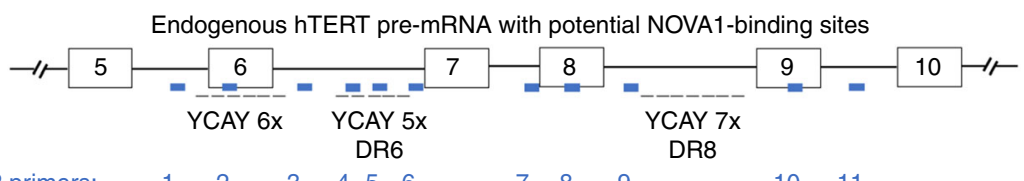

$\begin{array}{lllllllllllll}\text { UV-IP primers: } & 1 & 2 & 3 & 4 & 5 & 6 & 7 & 8 & 9 & & 10 & 11\end{array}$

C

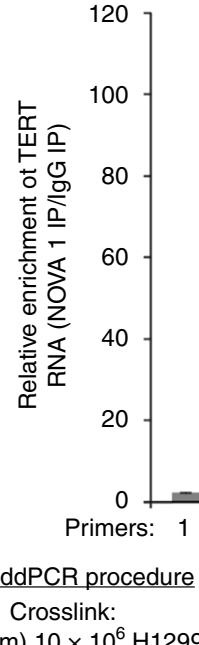

UV-C (254 nm) $10 \times 10^{6} \mathrm{H} 1299$ cells

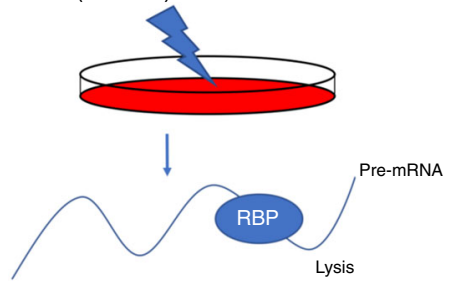

Treat with micrococcal nuclease

immunoprecipitation with NOVA 1 or IgG

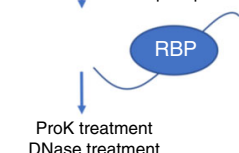

DNase treatment

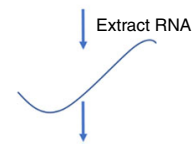

RT-ddPCR for TERT and other NOVA1 target RNAs

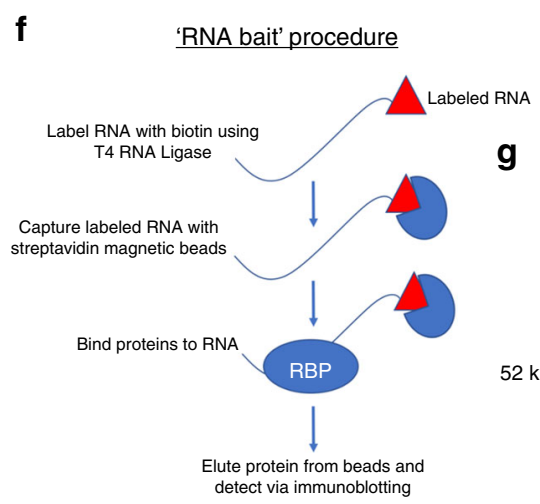

g
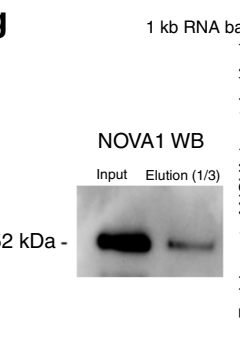

\section{e}

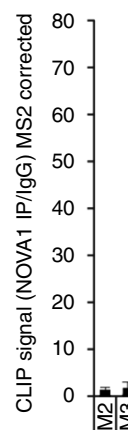

b UV-IP procedure

Crosslink:

UV-C (254 nm) $10 \times 10^{6} \mathrm{H} 1299$ cells

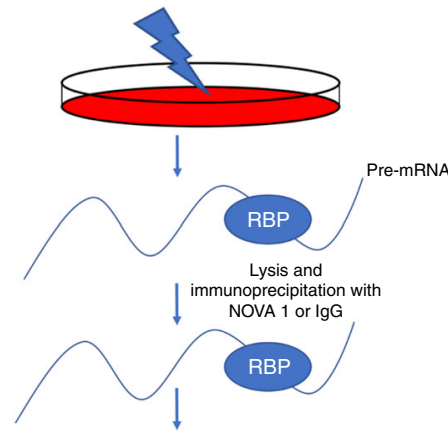

RNA extraction and RT-ddPCR

hTERT minigene pre-mRNA with potential NOVA1-binding sites and CLIP primers

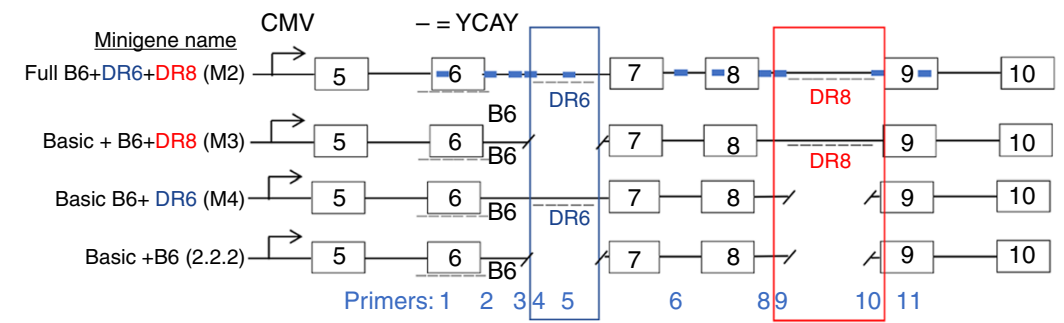

8. Since a $1 \mathrm{~kb}$ RNA fragment may bind many proteins, we generated a series of smaller ( $150 \mathrm{nt})$ RNA baits surrounding and in DR8 of $h T E R T$ to more specifically determine where NOVA1 was binding. We observed binding to oligos 3 , 4, and 5 (Fig. 4h) and weak to no binding to oligos 1 and 2 (Fig. 4h), with

the strongest binding to oligo 3 in the $5^{\prime}$ end of DR8. We hypothesize that NOVA1 is binding to DR8 and the region surrounding DR8 of $h T E R T$ through both direct and indirect interactions (that is, as part of a splicing factor complex) that directs NOVAl's impact on $h T E R T$ splicing. There are 7 YCAY 
Fig. 4 NOVA1 binds to a deep intronic element in TERT precursor RNAs. a Cartoon of TERT exons 5 through 10 showing potential NOVAT-binding sites and the primers (blue boxes) used in the ultraviolet immunoprecipitation (UV-IP) procedure. b UV-IP procedure schematic showing the major steps. $\mathbf{c}$ Droplet digital RT-PCR showing UV-IP enrichment of NOVA1 at TERT DR8 ( $n=3$ independent IPs). d Schematic of UV-crosslinking immunoprecipitation (CLIP) droplet digital PCR procedure. e Cartoon of hTERT minigenes used in the CLIP experiments, primers (light blue boxes), and droplet digital PCR quantification of NOVA1 protein and TERT RNA interaction ( $n=3$ independent IPs). Differences in constructs are highlighted in red and dark blue boxes and text. The minigenes where plasmid DNA was removed to generate the different constructs lacking the direct repeat regions are shown by a broken line. f Schematic of RNA bait procedure to find proteins that interact with $h T E R T$ RNAs. A second set of RNA baits were made from PCR fragments of $h T E R T$ intron 8 around and including DR8. $\mathbf{g}$ We in vitro transcribed a $1 \mathrm{~kb}$ fragment of $h T E R T$ intron 8 that contained DR8. Western blot and quantification of western showing pull down of NOVA1 protein with TERT RNA containing DR8 $(n=2)$. $\mathbf{h}$ Representative western blot of NOVA1 protein showing binding to DR8 at baits (oligos) 3,4 , and $5(n=2)$. Data are expressed as means and standard error of the mean where applicable. ${ }^{\star} p<0.05$. Supplementary data associated with this figure can be found in Supplementary Figure 4

motifs in DR8 (oligos 3 and 4), oligo 5, which is 71 nts $3^{\prime}$ of DR8 contains an additional 2 YCAY motifs. We hypothesize that a complex of proteins, including NOVA1, that interact at or near DR8 could be pulling down NOVA1 with oligo 5, explaining why NOVA1 is present in oligo 5's pull down. Overall, our results are consistent with the model that when NOVA1 is bound to hTERT mRNAs in intron 8 , it acts as a splicing enhancer, promoting the inclusion of exons 7 and 8 to increase the production of FL hTERT mRNAs.

Deletion or mutation of NOVA1 binding shifts hTERT splicing. To further define the importance of $h$ TERT DR8 in telomere biology, we deleted a 480-nt fragment of hTERT intron 8 using two CRISPR guide RNAs flanking DR8 (Fig. 5a). We identified three H1299 clones that had the correct on-target deletion of hTERT intron 8 DNA containing DR8 (Supplementary Figure $5 \mathrm{~A}, \mathrm{~B}$ and $\mathrm{C}$ ). In a parallel experiment, we introduced either wild-type (WT) or mutant DR8 DNA via a donor plasmid with CRISPR/Cas9 (Fig. 5b). The mutant donor plasmid had all seven of the YCAY motifs in DR8 mutated to YAAY, which has previously been shown to block NOVA1 recognition of target genes $^{34}$. We identified 5 clones with homozygous integration of the mutant NOVA1-binding sites and sorted an additional 6 single-cell WT clones to control for heterogeneity of tumor cell lines ${ }^{41}$ (Supplementary Figure 5E). This produced 15 cell lines (14 clones and the parental H1299 population) that we then followed over time for telomere length, telomerase activity, and TERT splicing phenotypes.

We observed clonal heterogeneity for telomere length between all the clones, including the control clones, as expected. In the sorted control clones, however, we observed minimal telomere length changes over time $(14 \pm 4.4$ nts per doubling; average \pm standard deviation), while significant telomere shortening was observed in both DR8 YAAY mutant clones (46 \pm 42 nts per doubling; $p=0.05)$ and in the DR8-deleted clones (108 $\pm 45 \mathrm{nts}$ per doubling; $p<0.001$ ) (Fig. 5c). All the DR8-deleted clones had longer telomeres on average compared to the mutant DR8 clones. Thus, deletion of DR8 may produce a strong selection pressure for clones with longer telomeres. hTERT splicing was also significantly changed, with a dramatic shift toward spliced products (35\% FL in sorted controls versus $12 \% \mathrm{FL}$ in DR8 YAAY mutants, $p<0.001$, Student's $t$; Fig. $5 \mathrm{~d}$, Supplementary Figure 5G). Further, we assayed telomerase enzyme activity over time at three different population doublings and observed that on average, the DR8 YAAY mutants had 70\% less telomerase activity compared to the controls while the DR8 deletion clones had nearly undetectable telomerase activity (Fig. 5f, Supplementary Figure $5 \mathrm{G})$. The telomere shortening rates of $46 \mathrm{nts}$ per doubling and 107 nts per cell doubling in the DR8 mutants $(70 \%$ telomerase inhibition) and DR8-deleted clones (nearly $100 \%$ telomerase inhibition) correlate closely with observations that telomerase adds 50-150 nts per cell division to maintain telomeres $^{42}$

We also observed clonal heterogeneity for $h T E R T$ splicing as shown in Fig. 5e: three clones had mostly minus beta, one clone had higher levels of hTERT FL and total hTERT mRNA, which correlated to higher telomerase activity (DR8 mutant 5), and one clone seemed to lack all transcripts with exons 5-9 (DR8 mutant 3, Supplementary Figure 5G and H). The clone (DR8 mutant 3) that completely lacked mRNA containing hTERT exons 5-9 also had no detectable telomerase activity (via TRAP) and eventually died in culture at population doubling 58 post sorting. Further, only one of three DR8-deleted clones showed FL hTERT message and telomerase activity (DR8 deletion 2, Supplementary Figure 5G and $\mathrm{H}$ ). After long-term passage one of the DR8 deletion clones stopped dividing at PD 85 (DR8 deletion 1). We measured the expression level of NOVA1 mRNA in these clones and observed that on average the clones were similar for NOVA1 mRNA expression levels (Supplementary Figure 5I), however we observed heterogeneity in expression between clones with different telomere biology phenotypes. For example, the deletion clone that died (DR8 deletion 1) and the mutant clone that died had low expression of NOVA1 compared to the average (Supplementary Figure 5). In contrast, the DR8 deletion clones with telomerase activity (DR8 deletion 2) had very high NOVA1 levels. This suggests that NOVA1 may help to maintain telomerase levels via an alternative binding site that promotes hTERT FL splicing. Overall, these data solidify the role of DR8 in the splicing choice of $h T E R T$ to produce FL or spliced products.

To determine if DR8 was important only in cell lines with NOVA1 or as a general splicing enhancer region, we compared the response of H1299 cells (cells that express NOVA1) and Calu6 cells (cells lacking NOVA1 expression) following treatment of an antisense oligonucleotide that blocks a sequence 19 nts from the $5^{\prime}$ end of DR8 (including a YCAY element, antisense oligo called, "DR8 + 19") and was previously shown to shift hTERT splicing toward minus beta containing transcripts in a Hela cell clone that expresses NOVA1 ${ }^{13}$. $\mathrm{H} 1299$ cells treated with $50 \mathrm{nM}$ of $\mathrm{DR} 8+19$ antisense oligo for $48 \mathrm{~h}$ significantly shifted $h T E R T$ splicing toward minus beta while Calu6 cells treated did not shift the splicing of hTERT (Supplementary Figure 5J and K). This indicates that DR8 is likely only an important splicing enhancer region in hTERT when NOVA1 is present.

\section{Discussion}

The catalytic protein component of telomerase, $h T E R T$, is spliced into multiple isoforms, but only FL $h T E R T$ mRNA is capable of producing enzymatically active protein that can maintain telomeres $^{13}$. We observed that $\sim 70 \%$ of the non-small cell lung cancers tested here express moderate to high levels of NOVA1. NOVA1 expression promotes the inclusion of exons 7 and 8 in the RT domain of $h T E R T$ to produce enzymatically active telomerase and regulate telomere length (Fig. 6). Further, NOVA1 
a

Endogenous hTERT locus showing DR8 deletion CRISPR/Cas9 strategy

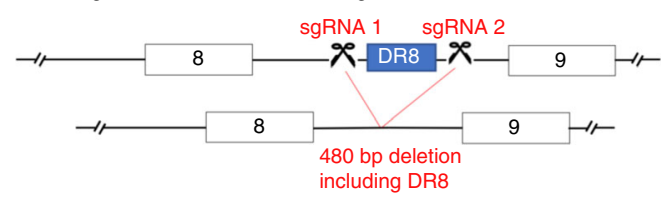

C b

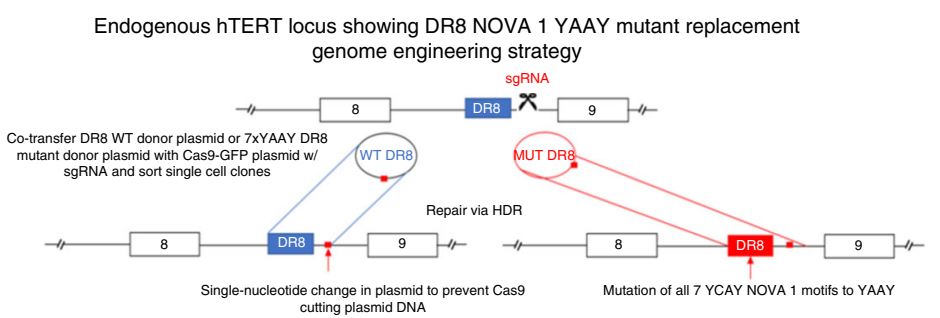

Telomere length (TRF) analysis of H1299 CRISPR/Cas9 clones and controls

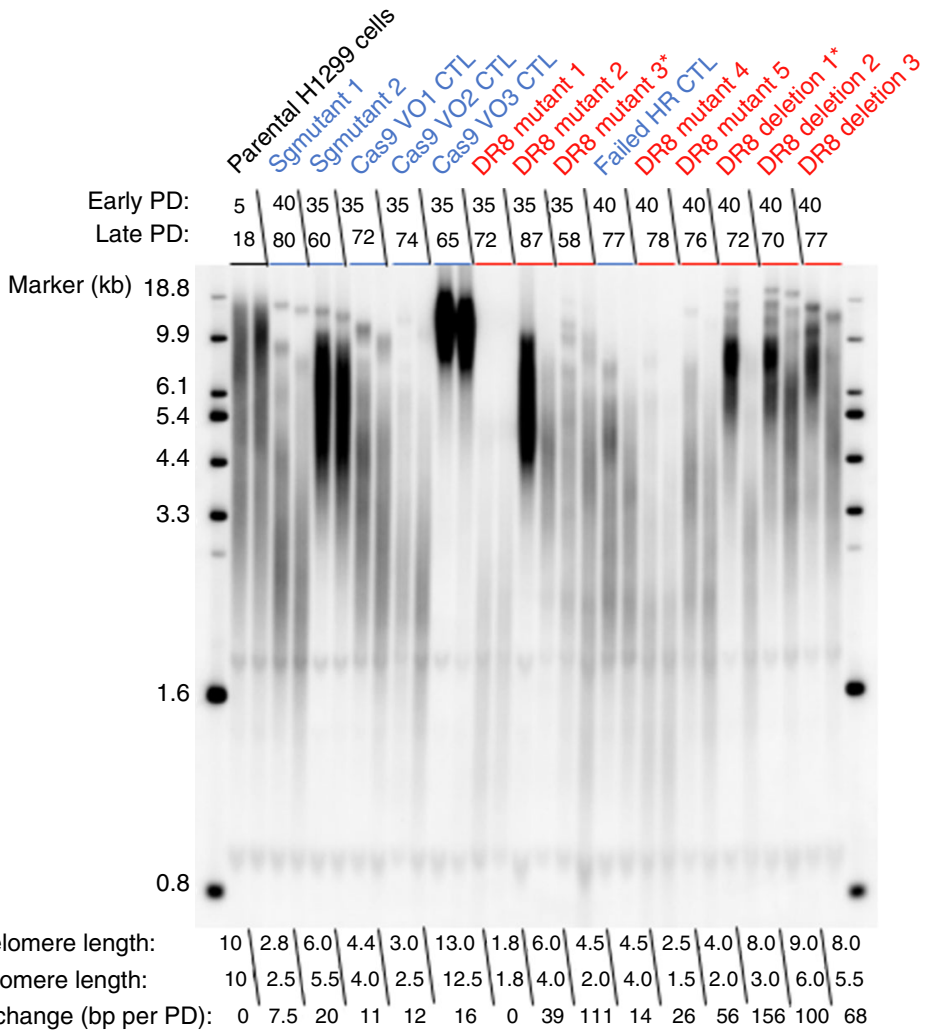

Early PD mean telomere length: Late PD mean telomere length: Telomere length change (bp per PD): \begin{tabular}{l|l|l|l|l|l|l|l|l|l|l|l|l|l|l}
10 & 2.5 & 5.5 & 4.0 & 2.5 & 12.5 & 1.8 & 4.0 & 2.0 & 4.0 & 1.5 & 2.0 & 3.0 & 6.0 & 5.5 \\
0 & 7.5 & 20 & 11 & 12 & 16 & 0 & 39 & 111 & 14 & 26 & 56 & 156 & 100 & 68
\end{tabular}

d

hTERT splicing shifts in mutant and deleted DR8 H1299 clones

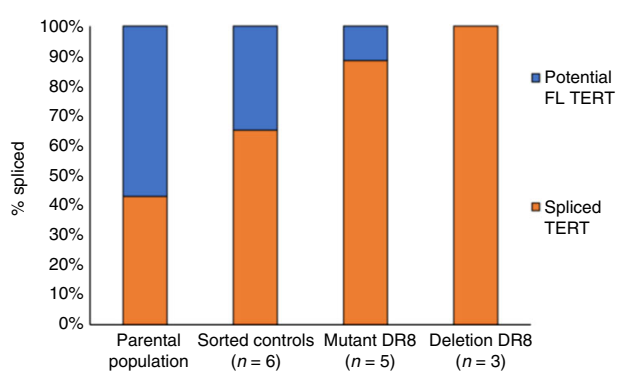

e Clonal heterogeneity of hTERT splicing in mutant and deleted DR8 $\mathrm{H} 1299$ clones

\section{f}

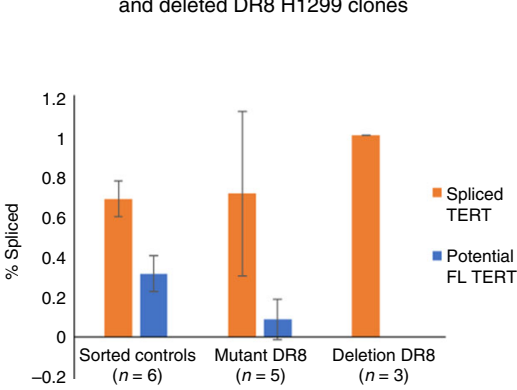

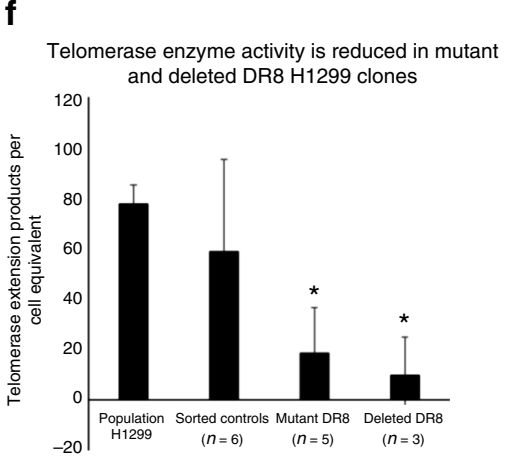

Fig. 5 Deletion of DR8 or mutation of NOVA1-binding sites in TERT shortens telomeres. a Schematic of CRISPR/Cas9 strategy to delete DR8 in TERT intron 8. b Schematic of CRISPR/Cas9 strategy to mutate NOVAT-binding sites in TERT DR8. c Terminal restriction fragment length (TRF-Southern blot) analysis of parental, sorted controls, DR8 NOVA1 '7 × YAAY' mutants, and DR8-deleted H1299 clones. *DR8 mutant 3 and *DR8 deletion 1 stopped growing at population doubling 57 and 85, respectively. d hTERT splicing isoform proportion analysis of parental, averaged sorted controls $(n=6)$, averaged DR8 mutants $(n=5)$, and averaged DR8 mutants $(n=3)$. e hTERT splicing isoform proportion analysis of averaged sorted controls $(n=6)$, averaged DR8 mutants $(n=5)$, and averaged DR8 mutants $(n=3)$. This plot shows the clonal heterogeneity of hTERT splicing in the clones used in this experiment. Standard deviation of the group means displays the variation in the response to mutation or deletion of direct repeat 8 (DR8) in hTERT. Only sorted controls and clones are shown as variation in the parental line is shown elsewhere. $\mathbf{f}$ Telomerase activity of parental, averaged controls $(n=6)$, averaged DR8 mutants $(n=5)$, and averaged DR8 deletion clones $(n=3)$. Data are expressed as means and standard deviation where applicable. ${ }^{*} p<0.05$. Supplementary data associated with this figure can be found in Supplementary Figure 5 
a

Model of $h T E R T$ splicing regulation by DR8 in NOVA1 + and NOVA1 - cells:

Model A - NOVA1 binding to DR8 acts as a splicing enhancer to include exons $7 / 8$, promoting the production of FL $h T E R T$ mRNAs

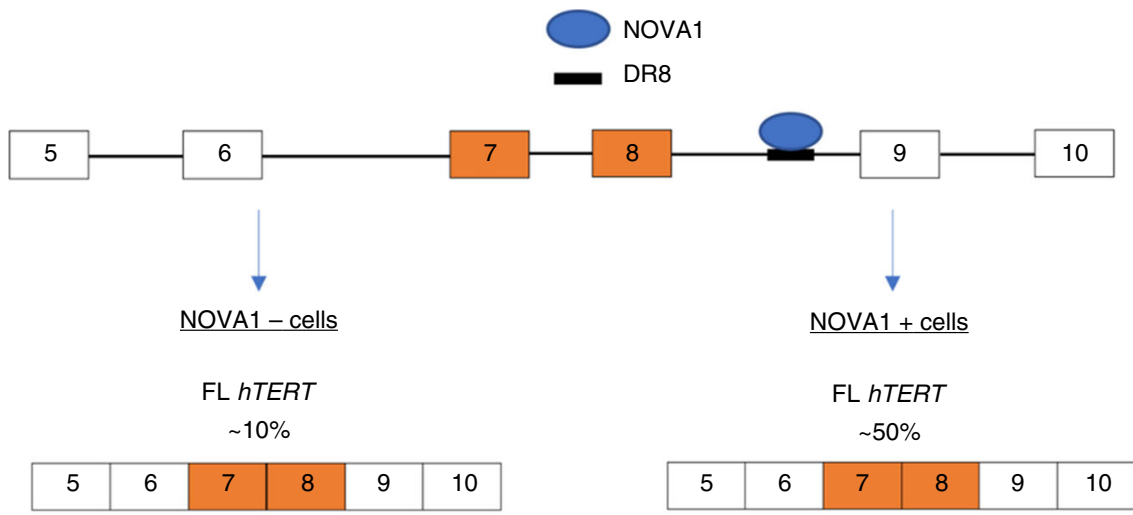

Spliced $h T E R T$

$\sim 90 \%$

\begin{tabular}{|l|l|l|l|}
\hline 5 & 6 & 9 & 10 \\
\hline
\end{tabular}

Spliced $h T E R T$

$\sim 50 \%$

\begin{tabular}{|l|l|l|l|}
\hline 5 & 6 & 9 & 10 \\
\hline
\end{tabular}

b

Model of $h T E R T$ splicing regulation by DR8 in NOVA1 + and NOVA1 - cells:

Model B - TERT RNA:RNA pairing of VNTR6 with complimentary sequence in intron 8 generates secondary structure favoring skipping of exons $7 / 8$ but NOVA1 disrupts this secondary structure and promotes inclusion of exons $7 / 8$

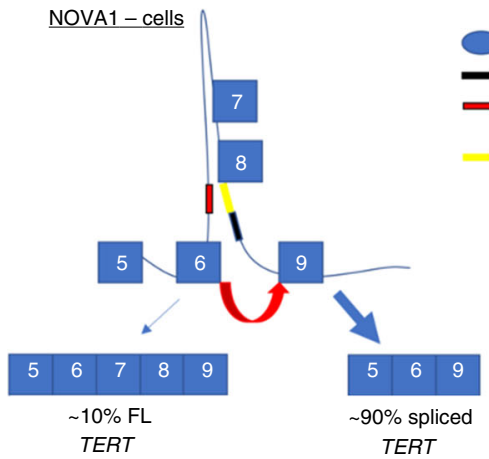

NOVA1 + cells

NOVA1
DR8
VNTR 6
repeats
Region in intron 8
complimentary to VNTR6

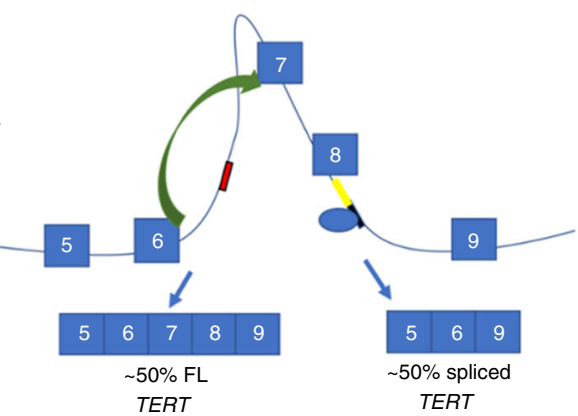

Fig. 6 NOVA1 binds to a deep intronic sequence in $h T E R T$ DR8 and acts as a splicing enhancer to promote the inclusion of exons $7 / 8$ and greater levels of full-length hTERT mRNAs. a Linear model depicting NOVA1 binding to TERT DR8 promoting the inclusion of exons 7/8. b Alternative model of NOVA1 promoting full-length TERT considering RNA:RNA pairing/RNA secondary structure of TERT pre-mRNAs

may be a regulator of growth and invasion-related signaling in cancer cells, coupling telomere length maintenance to other cancer cell characteristics (Fig. 3). Thus, the applicability of the hTERT minigene screen data we have compiled represents a valuable resource that could be mined to identify other splicing factors that could be targeted in cancer.

These studies extend earlier findings that RNA metabolism is a regulatory component of telomerase activity and telomere length maintenance ${ }^{43,44}$. For instance, the mRNA decay pathway was found to be critical in telomere length maintenance in yeast ${ }^{45}$. Additionally, our splicing factor screen and the work of others point to the importance of hnRNP proteins in telomere biology and in the regulation of $h$ TERT pre-mRNA splicing ${ }^{46-51}$ (Fig. 1). We confirmed factors that were previously reported to directly bind to $h T E R T$ pre-mRNA and regulate minus beta splice choice (SRSF11, hnRNPH2, and hnRNPL) ${ }^{15}$. We observed significant changes in the minigene reporter assay for hnRNPH1, hnRNPF, and hnRNPM, which were all observed to potentially bind $h T E R T$ pre-mRNAs in CLIP experiments ${ }^{52}$, further validating the minigene data. As far as we could determine, this is the first study to investigate knockdown of splicing factors in human cancer cells with regard to telomere biology and we observed that even modest knockdown is compatible with long-term tissue culture studies to investigate changes in the telomere length of cells.

During the generation of CRISPR/Cas9 clones of H1299 lung cancer cells some striking and unexpected phenotypes arose upon mutation and deletion of DR8 in hTERT. Two clones that eventually died off in culture had about $2 \mathrm{~kb}$ telomere lengths when they died (DR8 mutant clone 3 and DR8 deletion clone 1). However, DR8 mutant clone 4 had a mean telomere length of about $2 \mathrm{~kb}$ and survived in culture (Fig. 5). We speculate that the telomere DNA damage sensing capabilities between the clones that died compared to the clones that survived at these short telomere lengths are different. Further, DR8 mutant clone 3 and DR8 deletion clone 1 both lacked expression of $h T E R T$ exons 5-9, while DR8 mutant clone 4 had a small amount of FL hTERT remaining (Fig. 5). This small amount of hTERT FL could have generated enough active telomerase to maintain the short telomeres. We speculate DR8 mutant clone 4 could have had recruitment and processivity advantages compared to other lines 
with higher telomerase activity but increased rates of telomere shortening. These clones could be useful in future studies aiming at elucidating the genetic and molecular underpinnings of these less-well-described phenomena.

We propose two models for how the NOVA1 protein is interacting with TERT pre-mRNAs (Fig. 6). In the first model only considering NOVA1 protein's influence on hTERT premRNAs, we propose that NOVA1 binds to DR8, recruiting the basal exon junction recognition machinery that promotes the use of exon 8's 5' splice donor with exon 9's 3' splice acceptor site to generate $h T E R T$ mRNAs, including exons 7 and 8 (Fig. 6a). In the second model, we consider both pre-mRNA secondary structures and NOVA1 proteins. We previously defined a role for RNA: RNA pairing in regulating minus beta splice choice ${ }^{14}$. In this model (Fig. 6b), RNA:RNA pairing of a variable nucleotide tandem repeat in intron 6 of $h T E R T$ creates an RNA secondary structure that brings the exon $65^{\prime}$ splice site in close proximity to the exon $93^{\prime}$ splice site, generating a structural change where joining of exon 6 to exon 9 is favored over exon 6 to exon 7 , producing the minus beta splice variant over FL mRNAs. When NOVA1 is present and bound to DR8, this disrupts the secondary structure created by RNA:RNA pairing between intron 6 and intron 8 and brings the exon $85^{\prime}$ splice site closer to the exon $93^{\prime}$ splice site thus producing more FL molecules of $h T E R T$ compared to cells lacking NOVA1 (Fig. 6b). Supporting this model, when DR8 is deleted or all seven YCAY motifs in DR8 are mutated three lines switched their splicing to almost solely minus beta (Supplementary Figure 5). In one DR8 mutant (clone 3) and one DR8 deletion (clone 1) we observed a complete loss of expression of $h T E R T$ exons 5-9 (Supplementary Figure 5). One possibility is that the loss of NOVA1 binding in these clones leads to a change in secondary structure in the pre-mRNA of $h T E R T$ and results in a complete skipping of exons 5-9 but that other regions of $h T E R T$ are still expressed (Supplementary Figure 5H).

The present studies add to the growing body of literature that alternative splicing may be targetable in cancer $^{53}$. The present study points out that telomerase activity and telomere length maintenance can be manipulated by targeting the alternative splicing machinery. We chose to follow-up on NOVA1, a well-defined splicing factor that has implications in cancer biology as well, because it had binding sites in $h T E R T$ introns and exons, and because it displays cancer cell-specific expression across a wide variety of human tissues (Fig. 1, besides neurons and testis). In addition to NOVAl's role in neurons, NOVA1 can also have a role in the survival of cancer cells (Fig. 3). It is unlikely that NOVA2 is playing a similar role in cancer biology as it is only expressed in brain cancers and absent in nearly all other human tumors (http://www.proteinatlas. org/ENSG00000104967-NOVA2/cancer).

It is clear that NOVA1 has additional targets in cancer cells that are related to the growth phenotypes observed herein. NOVA1 may regulate AKT levels influencing growth and survival of cancer cells. The role of AKT in cell proliferation and survival is well documented and several anticancer drugs are currently in use that target AKT. Further, AKT is upstream of FOXO transcription factors, which controls cell survival and apoptosis. AKT functions in cellular survival by phosphorylating and sequestering FOXO's in the cytoplasm and keeping them inactive and unable to initiate transcription of the cell death program. When NOVA1 levels are reduced, AKT protein levels are reduced ${ }^{32}$, FOXO's become active and initiate transcription of the cellular death machinery. Future studies investigating the role of NOVA1 in AKT induced cancer cell survival are warranted. Further, AKT is also a kinase that may increase the nuclear pool of TERT proteins and thus increase the potential for active telomere length maintaining telomerase ${ }^{54}$. Thus, a NOVA1-AKT-TERT axis may exist in some cancers that may be targetable.
Overall, our results are consistent with the idea that hTERT splicing is malleable in cancer cells by either targeting the intronic DR8 region or by targeting NOVA1. While NOVA1 impacts hTERT pre-mRNAs, it also has other targets in cancer cells. Further studies will be required to determine if NOVA1 is indeed clinically targetable in cancer, including the identification of proteins that interact with NOVA1 and the genes that NOVA1 regulates in cancer. In summary, NOVA1 appears to integrate replicative immortality (telomerase activity and telomere length maintenance), with sustained proliferative signaling, invasion, and metastasis. Thus, the present studies have provided new insights into $h T E R T$ regulation and identified NOVA1 as a lead candidate gene for development of small-molecule inhibitors targeting the splicing machinery for anticancer therapy.

\section{Methods}

Plasmids. Human telomerase ( $h T E R T)$ exon and intron sequences were inserted into pcDNA5/FRT expression vector (Invitrogen, Carlbad, CA) and were modified to exclude exons 5 and 10 and introns 5 and 9 to shorten the construct ${ }^{13}$. The hTERT minigene was modified with renilla and firefly luciferase fragments. The minigene was integrated into a single locus using previously described methods ${ }^{13,14}$. We modified the previously used minigene to include a renilla $(R l u c)$ and a firefly luciferase $(F l u c)$ gene. We fused renilla luciferase and E2A (equine rhinitis A virus 2A) peptide in frame to $h T E R T$ exon 8 so that when exon 8 was included in the minigene pre-mRNA, the protein product would produce Rluc (Fig. 1a) putting the Fluc out of frame. Conversely, when exons 7 and 8 are skipped and splicing occurs from exon 6 to exon 9, Fluc and a T2A (Thosea asigna virus 2A) peptide are in frame. The use of the $2 \mathrm{~A}$ peptides facilitated the generation of equal molar ratios of luciferase to $h T E R T^{55}$. Lentiviral plasmid for secreted embryonic alkaline phosphatase (SEAP, Clontech) Lentiviral GFP pGIPZ shRNA plasmids for control (non-targeting), NOVA1 (Openbiosystems, NOVA1-5'-TTGGACTTAGACAG CTTGA) were obtained. Lentivirus was made by co-transfecting $5 \mu \mathrm{g}$ of proviral shRNA plasmids and $2 \mu \mathrm{g}$ of packaging plasmids pMD2.G and psPAX2 using Polyjet transfection reagent (SignaGen Laboratories) into 293FT cells. CCSB-Broad lentiviral human NOVA1 FL cDNA with a C-terminal V5 tag and blasticidin selection in mammalian cells (accession: BC075038, clone ID: ccsbBroad304_01104) was purchased and sequence verified by our group (GE, Dharmacon). Viral particles were produced as above. ShRNA-resistant NOVA1 cDNA was generated by site-directed mutagenesis (Agilent Quickchange). Retroviral particles were generated in 293FT packaging cells with pUMVC and pVSVG Plasmids for CRISPR/Cas9 experiments are described below in the genome engineering section.

Cell culture and cell lines. All non-small cell lung cancer cell lines (H1299, H920, Calu6, and $\mathrm{H} 2882$ ) were cultured at $37^{\circ} \mathrm{C}$ in $5 \% \mathrm{CO}_{2}$ in 4:1 DMEM:Medium 199 containing $10 \%$ calf serum (HyClone, Logan, UT). Briefly, HBECs were maintained in low oxygen conditions in serum-free media containing supplements from the Keratinocyte-SFM media (Invitrogen/Gibco catalog \# 17005-42) on a collagen/ gelatin-coated tissue culture dish ${ }^{56}$. All cell lines were obtained from American Type Culture Collection, or as a kind gift from Drs. John Minna and Adi Gazdar.

Cancer growth assays. Stable knockdown cells and controls (non-targeting shRNA) were suspended in $0.375 \%$ Noble agar (Difcon, Detroit) in supplemented basal medium at two densities (1000 and 2000 cells) and overlaid on $0.75 \%$ Noble agar in 24-well plates. Each density was seeded in triplicate and each assay was performed twice. Colony formation efficiency was calculated by the average number of colonies counted per cell divided by the number of cells seeded. Colonies larger than $0.1 \mathrm{~mm}$ were measured and counted after 10 days of growth and the average of the counts was used. Data are plotted as fold change over nontargeting shRNA cells. Data were analyzed with two-tailed Student's $t$ tests ${ }^{57}$.

Invasion was determined using Boyden chamber assays. Briefly, cells were serum starved overnight $(\sim 16 \mathrm{~h})$ prior to assays for invasion. Twenty-four-well Matrigel-coated transwell filters (BD Biosciences, San Jose, CA) were thawed and rehydrated according to the manufacturer's instructions. Cells were collected and re-suspended in serum-free media and added to the top chamber in duplicate. The bottom chamber was filled with $2 \%$ serum-containing media (4:1 DMEM:Medium 199 ) as a chemoattractant. Cells were incubated overnight. Non-invaded cells were scraped off with a cotton swab and wells were washed with phosphate-buffered saline (PBS). Invaded cells were fixed for $5 \mathrm{~min}$ with $10 \%$ neutral buffered formalin and stained for $10 \mathrm{~min}$ with Hoechst (Invitrogen). Images were taken at $\times 10$ magnification ${ }^{58}$.

For colony formation assays, cells were plated at clonal density (30-70 cells per $2.5 \mathrm{~cm}^{2}$ dish) in $10 \%$ serum-containing media. Cells were analyzed 7 days after plating by staining with Hoechst (Invitrogen $)^{59}$.

To test the dependence of observed growth defects on telomere length and telomerase activity, an $h T E R T$ cDNA (pMIN-Ub-IRES-Blast lentiviral vector from 
ref. ${ }^{36}$ ) was transdued into H1299 cells with control shRNAs or NOVA1 shRNA after 50 population doublings. This vector contains a $3.398 \mathrm{~kb}$ cDNA of $h T E R T$ coding sequence thus no splicing is needed to make hTERT mRNA. Further information about the hTERT cDNA from pGRN145 that was inserted into a lentiviral vector can be found here (https://www.atcc.org/Products/All/MBA-141. aspx\#generalinformation). It is clear that TERT RT domain was expressed in our experiments and that telomerase was active (see Supplementary Figure 5). Following infection and selection, cells were plated as described above in the cancer growth assays.

Xenograft. All animal experiments were approved by the University of Texas Southwestern (UTSW) Institutional Animal Care and Use Committee (IACUC) and conducted as per the institutional guidelines. Athymic NRC nu/nu nude mice ( 4-6 weeks old, Charles River) were purchased. Tissue culture cells from H920 control shRNA, H920 NOVA1 shRNA, Calu6 control (WT with empty lentiviral vector), and Calu6 plus NOVA1 cDNA were cultured and injected subcutaneously into the hind flanks. For H920 cells 5 million cells in $100 \mu \mathrm{L}$ of $1 \times$ PBS were injected. For Calu6 cell 1 million cells in $100 \mu \mathrm{L}$ of $1 \times$ PBS were injected. Tumor growth was monitored by caliper measurement once or twice weekly. Tumor volume was calculated $\left(\right.$ volume $=(\text { width })^{2} \times$ length $\left./ 2\right)$.

Transient siRNA experiments. For transient knockdown experiments cells were plated in six-well plates (150000 cells per well) and were transfected with nonsilencing controls (Santa Cruz Biotechnology, sc-37007) or a pool of three siRNAs targeting NOVA1 (Santa Cruz Biotechnology, sc-42142: sense RNA sequences-(1) 5'-GACAGACAAUUGUUCAGUUtt-3', (2) 5'-GAACGGUUGAAGCACUGAAtt-3', (3) 5'-GACCACCGUUAAUCCAGAUtt-3'). Cells were plated $24 \mathrm{~h}$ prior to transfections. On the day of transfection, media was switched to $2 \%$ serum and transfection complexes were prepared with $50 \mathrm{nM}$ of siRNAs using MEM (Gibco, Invitrogen) and RNAi max (Invitrogen) following the manufacturer's procedures. Following $72 \mathrm{~h}$ of exposure to siRNAs cells were washed, trypsinized, counted, and pelleted for RNA extraction and telomerase activity assays. We treated normal BJ fibroblasts three times (every $96 \mathrm{~h}$ ) with non-targeting control or NOVA1 targeting siRNAs over the course of 12 days, counted the cells, and determined viability with trypan blue staining.

Western blot analysis. Total protein lysates were extracted from tissue culture cells using Laemmli buffer, boiled, and the protein concentration determined (BCA protein assay, Pierce). Thirty micrograms of protein was resolved on SDSpolyacrylamide gel electrophoresis gels, transferred to polyvinylidene fluoride membranes, and detected with a rabbit monoclonal antibody for NOVA1 (Abcam, EPR13847, ab183024, 1:1000 dilution in 5\%BSA)). Protein loading was determined with antibodies against with beta actin (Sigma; 1: 20000 dilution in 5\% non-fat dry milk) or histone H3 (Sigma; 1:1000 in 5\% non-fat dry milk). Uncropped images of all western blots can be found in Supplementary Figure 7.

RT-droplet digital PCR. Tissue panel RNAs were purchased (Clontech, 20 tissue panel II). Three sets of CDNAs were made with a 1:1 mixture of random hexamer and oligo-dT priming with three different RTs: (1) iScript advanced $\left(42^{\circ} \mathrm{C}\right.$, BioRad); (2) Superscript III $\left(55^{\circ} \mathrm{C}\right.$, Invitrogen); and (3) AMV ( $50^{\circ} \mathrm{C}$, Invitrogen). All RNA samples were spiked with a known amount of MS2 bacteriophage RNA to enable normalization of absolute molecule counts from ddPCR. For tissue panel $h T E R T$ and NOVA1 mRNA analysis we used three RTs because we observed differences in detection of $h T E R T$ using different RTs so to be able to eliminate spurious measures of low abundance targets we averaged data for all three RTs. All cDNAs were diluted 1:4 before use and stored at $-80^{\circ} \mathrm{C}$. For $h T E R T$ splicing analyses we used iScript Advanced (Bio-Rad) to generate cDNAs, diluted 1:4, and used within $48 \mathrm{~h}$ of production in ddPCR measures. Primer sequences for TERT are listed in Supplementary Table 2. Uncropped gel images of RT-PCR gels can be found in Supplementary Figure 7.

Droplet digital TRAP assay (telomerase activity). Quantitation of telomerase enzyme activity was performed using a modified telomeric repeat amplification protocol ${ }^{23}$. Briefly, cells were lysed, diluted, and added to the telomerase extension reaction for $40 \mathrm{~min}$ followed by heat inactivation of telomerase. An aliquot of the extension products was amplified in a ddPCR for 40 cycles and fluorescence measured and droplets read and counted on the droplet reader (QX200, Bio-Rad). Following, data were processed and telomerase extension products per cell equivalents determined.

Telomere length analysis. The average length of telomeres (terminal restriction fragment lengths) was measured as described in ref. ${ }^{60}$ with the following modifications. DNA was transferred to Hybond-N+ membranes (GE Healthcare, Piscataway, NJ) using vacuum transfer. The membrane was briefly air-dried and DNA was fixed by UV-crosslinking. Membranes were then probed for telomeres using a digoxigenin (DIG)-labeled telomere probe, detected with an horeseradish peroxidase-linked anti-DIG antibody (Roche), and exposed with CDP-star
(Roche $)^{61}$. Uncropped scans of Southern blot images can be found in Supplementary Figure 7.

Minigene screen setup and reporter assays. We used two databases (NCBI gene and Genecards) and searched the key words of "RNA binding protein" and "splicing factor" to generate a list of 516 genes (Supplementary Data file 1) and then ordered pools of four siRNAs to each gene (Dharmacon, GE, sequences of pools to each gene are located in Supplementary Data file 1, we had 528 pools of siRNA as several genes had multiple siRNA pools). HeLa cells harboring the $h T E R T$ minigene splicing reporter were plated and transfected $24 \mathrm{~h}$ later with $1 \mathrm{nM}$ of each individual pool of siRNAs. A low concentration of siRNAs ( $1 \mathrm{nM})$ helped to reduce the potential viability effects and "off-target" effects. Plates were transfected using RNAi max (Invitrogen; sequences in Supplementary Data file 1) and cells were analyzed $72 \mathrm{~h}$ following transfection. As negative controls, we used a pool of scrambled siRNAs (siRNA control), a transfection control (cells, transfection reagents, and media), and a "cells only" control (cells plus culture media).

Knockdown of the core splicing factor $h n R N P H 1$ resulted in a fourfold induction of minus beta splicing in the minigene luciferase assay $(p<0.05$, Student's $t$; Supplementary Figure 1A). $h n R N P H 1$ was included as a minus beta inducing positive control and ubiquitin $(U B B)$ as a transfection-positive control. Cells were lysed in passive lysis buffer and analyzed for renillia and firefly luciferase following the manufacturer's instructions after $72 \mathrm{~h}$ (Dual-luciferase reporter assay system, Promega). The screen was repeated twice and data were averaged for each luciferase measurement. Then, a ratio of minus beta to FL splice variants were calculated for each target gene. Each ratio was then expressed relative to the ratio of the siRNA control (set to a value of 1). Since many RNA-processing factors have documented effects on cell viability, we also infected cells with a SEAP reporter lentivirus. Only living cells will produce and secrete SEAP. Thus, we used SEAP as a viability control (Supplementary Figure 1B). Conditioned media $(20 \mu \mathrm{L}$ from the siRNA-transfected cells) was analyzed for SEAP (Great EscAPe SEAP Chemiluminescence kit). As shown in Supplementary Figure 1B, siRNA depletion of UBB resulted in a loss of cell viability compared to the "cells only" condition. SEAP data were used following identification of a splicing hit to determine if the change in $h T E R T$ splicing reporter ratio was due to changes in cell viability.

Ultraviolet immunoprecipitation RT-PCR. UV-IP was performed as described ${ }^{38}$ with slight modifications. Briefly, 90\% confluent cells were crosslinked with UV-C $\left(254 \mathrm{~nm}, 250 \mathrm{mj} \mathrm{cm}^{-2}\right)$, scrapped, washed, and pelleted. Pellets were then lysed in RIPA buffer containing an RNase inhibitor (Ambion), mixed with antibodies conjugated to magnetic beads (rabbit IgG or rabbit monoclonal NOVA1, Abcam, EPR13847, ab183024), and washed. RNA was extracted and RT-ddPCR performed with a series of primers designed near and around the in silico-identified potential NOVA1-binding sites (Fig. 4a, b).

Crosslinking immunoprecipitation RT-ddPCR. To perform CLIP we UV (UV-C, $254 \mathrm{~nm}, 250 \mathrm{mj} \mathrm{cm}^{-2}$ )-crosslinked cells in $15 \mathrm{~cm}$ dishes with $3 \mathrm{~mL} 1 \times$ PBS. A volume of $7 \mathrm{~mL}$ of ice-cold $1 \times$ PBS was added to the crosslinked cells and the cells were scrapped and collected by centrifugation $\left(4^{\circ} \mathrm{C}, 0.2 \times g\right.$ for $\left.5 \mathrm{~min}\right)$. Supernatant was removed and $1 \mathrm{~mL}$ of ice-cold $1 \times$ PBS was added and the cells spun and pelleted a second time in a $1.5 \mathrm{~mL}$ microcentrifuge tube. The cells were lysed in $0.5 \% \mathrm{SDS}$ lysis buffer (Tris-HCl, pH 7, $1 \mathrm{mM}$ EDTA, $1 \mathrm{mM}$ dithiothreitol, RNase inhibitor (Ambion), and $1 \mathrm{mM}$ phenylmethylsulfonyl fluoride; $140 \mu \mathrm{L}$ per sample) and heated to $65^{\circ} \mathrm{C}$ for $5 \mathrm{~min}$ and immediately placed on ice for $5 \mathrm{~min}$. The lysate was volume corrected to $700 \mu \mathrm{L}$ in RIPA correction buffer $(1.25 \% \mathrm{NP} 40,0.625 \%$ sodium deoxycholate, $62.5 \mathrm{mM}$ Tris-HCl, pH 8, $2.25 \mathrm{mM}$ EDTA, $187.5 \mathrm{mM} \mathrm{NaCl}$, RNase inhibitor (Ambion), and $1 \mathrm{mM}$ phenylmethylsulfonyl fluoride; $560 \mu \mathrm{L}$ per sample). The lysate $(700 \mu \mathrm{L})$ was then passed over a Qiashredder ${ }^{\circ}$ column twice (centrifuged for $30 \mathrm{~s}$ at $13000 \times g$ at $4^{\circ} \mathrm{C}$ ). The entire lysate was then centrifuged for $15 \mathrm{~min}$ at $16000 \times g$ at $4^{\circ} \mathrm{C}$ and the supernatant transferred to a new microcentrifuge tube. The lysates were then treated with micrococcal nuclease $(0.15 \mathrm{U}$ in $50 \mathrm{mM} \mathrm{CaCl}$ buffer for $10 \mathrm{~min}$ at $37^{\circ} \mathrm{C}$ ), immediately placed on ice, and $20 \mathrm{mM}$ EGTA added to quench the micrococcal nuclease activity. Antibodies and beads were prepared at room temperature in sodium phosphate buffer $(0.1 \mathrm{M}, \mathrm{pH}$ 8.1; Anti-Noval antibody [EPR13847] (ab183024), Abcam; Rabbit IgG) and mixed with magnetic protein A/G beads (Dynabeads, $50 \mu \mathrm{L}$ per sample) and added to the cleared lysate. The samples (NOVA1 or IgG) were immunoprecipitated at $4{ }^{\circ} \mathrm{C}$ for $4 \mathrm{~h}$. The samples were then washed five times in RIPA buffer ( $1 \%$ NP40, $0.5 \%$ sodium deoxycholate, $0.1 \%$ SDS, $150 \mathrm{mM} \mathrm{NaCl}$, Tris- $\mathrm{HCl}, \mathrm{pH} 8$, and $2 \mathrm{mM}$ EDTA). After the final wash the beadprotein-RNA complexes were re-suspended in $200 \mu \mathrm{L}$ of RIPA buffer and $2 \mathrm{U}$ of RNase-free DNase (Ambion) was added in $300 \mu \mathrm{L}$ of DNase buffer and incubated for $10 \mathrm{~min}$ at $37^{\circ} \mathrm{C}$ with gentle agitation. Beads were collected with the magnet and supernatant removed. Proteinase $\mathrm{K}$ buffer was added $\left(0.5 \mathrm{mg} \mathrm{mL}^{-1}\right.$ proteinase $\mathrm{K}$, $0.5 \%$ SDS, $20 \mathrm{mM}$ Tris-HCl, pH 7.5, $5 \mathrm{mM}$ EDTA, 8 ng of MS2 RNA, and $5 \mu \mathrm{L}$ of MRC RNA precipitation carrier; $300 \mu \mathrm{L}$ per sample) and incubated at $37^{\circ} \mathrm{C}$ for $15 \mathrm{~min}$ with shaking. RNA was then precipitated with sodium acetate and phenol chloroform. Following RNA precipitation reverse transcription was performed with Superscirpt III (Invitrogen) with random hexamers. ddPCR was performed with Evagreen ${ }^{\text {. }}$. 
RNA pull down with biotinylated RNA baits. A plasmid was generated (TOPO TA) via PCR from a BAC containing $h T E R T$ (RP11-990A6, CHORI) using primers that generated a $1 \mathrm{~kb}$ fragment of $h T E R T$ intron 8 including DR8. Following integration into the TOPO TA vector, in vitro transcription was performed using the T7 promoter (Ampliscribe T7 kit, Ambion, Life technologies) following the manufacturer's instructions, including a $45 \mathrm{~min}$ DNase step prior to RNA precipitation. RNA was isolated and biotinylated at the $3^{\prime}$ end (Pierce RNA $3^{\prime}$ end biotinylation kit). Biotinylated RNA was purified with streptavidin beads. Cell lysates were prepared following the kit instructions (Peirce Magnetic RNA-protein pull-down kit). Protein-RNA complexes were immunoblotted for NOVAl following pull down. To produce the smaller RNA baits, T7 promoter sequences were incorporated into the $5^{\prime}$ end of the forward primers of each region of interest in and surrounding $h T E R T$ DR8. The same procedure was followed as above to generate the RNA baits. In both cases 293FT cells were transfected with V5-tagged NOVA1 cDNA construct using lipofectamine 2000. After $48 \mathrm{~h}$, triplicate samples of $10 \times 10^{6}$ cell were washed, typisinized, counted, pelleted, and frozen at $-80^{\circ} \mathrm{C}$ until analysis.

Genome-editing and engineering methods (CRISPR/Cas9 methods). To delete $h T E R T$ DR8 we designed two guide RNAs (pre-DR8 guide- $5^{\prime}$-ATCTGCTTGC GTTGACTCGC-3' and post DR8: 5' $^{\prime}$-TTATTTTCGGGAAGCGCTAT-3') and cloned these guides into PX458 (Addgene Plasmid \#48138-pSpCas9(BB)-2AGFP). Cells were transfected using Lipofectamine $3000^{\circ}$ following the manufacturer's instructions for scaling up to $10 \mathrm{~cm}$ dish. Forty-eight hours after transfection cells were flow sorted for the top 5\% green fluorescent protein (GFP)positive cells into individual wells of a 96 -well plate. After about 14 days wells with growing cells were scaled up to a six-well plate and once confluent scaled up to a $10 \mathrm{~cm}$ dish. Cells were collected for DNA extraction and genotype analysis of CRISPR mutation validation. To validate the deletion the 480-base pair sequence containing DR8 of $h T E R T$, we performed PCR with two different primer sets of different sizes to ensure our results were robust. We also isolated the PCR product from primer set two and performed Sanger sequencing to verify the recombination event was between two TERT alleles and not a different sequence in the genome.

To mutate endogenous $h T E R T$ we had two plasmids synthesized (SGI-DNA). The WT plasmid (WT- $h$ TERT intron 8 mutant PAM in pUC-SGI) contained 2000 bases of $h T E R T$ intron 8 surrounding DR8 that had the PAM sequence of the postDR8 guide above mutated from $5^{\prime}$-CCT to ACT. The mutant plasmid (MUT$7 \times$ "YAAY" DR8 MUT $h T E R T$ mutant PAM in pUC-SGI) was identical to the WT plasmid except that we mutated all seven of the "YCAY" motifs in DR8 to "YAAY" (changing the central CA to AA is known to block NOVA1 recognition ${ }^{34}$ ). We then co-transfected the guide RNA post DR8 and either WT or MUT plasmid in the presence or absence of NHEJ inhibitor SRC7-pyrazine. We pre-treated cells with $50 \mathrm{nM}$ SCR7 for $30 \mathrm{~min}$ prior to transfection. Cells were transfected with Lipofectamine $3000^{\circ}$ and $48 \mathrm{~h}$ later the top 5\% GFP-positive cells were sorted as above. Once wells with growing cells were identified we scaled up the clones as above. We isolated DNA from the clones to validate the insertion of WT or MUT plasmids. To validate WT insertion clones, we used PCR to amplify a sequence surrounding DR8 and purified and sequenced the PCR product via Sanger sequencing. To screen and validate MUT clones, we used PCR to amplify a region around DR8 and then digested the DNA with BclI. Conveniently, the mutations in DR8 (YCAY sites 4 and 5) introduced a novel restriction enzyme site that allowed us to identify mutant clones via PCR and REN digestion (similar to RFLP analysis, Supplementary Figure 5D, E, and F). We also Sanger sequence validated the MUT clones. We were able to validate two WT DR8 clones with homozygous PAM sequence mutation, indicating that homologous recombination of the donor plasmid was successful. We were also able to validate that 5 clones had homozygous integration of mutant DR8. We also sorted a variety of controls (vector only and one control exposed to both Cas9 and mutant donor that failed to undergo cutting and homology directed (HR) repair, "failed HR control") since it is well established that clonal heterogeneity exists in tumor cells lines ${ }^{41}$. These clones were all validated by PCR and Sanger sequencing to have WT DR8 $h T E R T$ sequences (representative Sanger sequencing in Supplementary Figure 5E). This produced 15 cell lines (14 clones and the parental H1299 population) that we then followed over time for telomere length, telomerase activity, and TERT splicing phenotypes.

Study approval. All animal experiments were approved by the UTSW IACUC and conducted as per institutional guidelines.

Statistics. Unless otherwise noted in the Methods section, figure legend, or in the Results section, pairwise Student's $t$ tests (two-sided) were used to determine statistically significant differences between group means. Significant differences were accepted at a $p$ value $<0.05$. For analysis of microarray data, we correlated the expression data of 528 RNA-binding proteins across six cell lines (differential expression analysis ( $t$ tests) and false-discovery rate corrected with BenjaminiHochberg procedure).

Data availability. The datasets generated during and/or analyzed during the current study are available in the GEO repository under accession number
GSE32036. All other data that support the findings of this study are available from the corresponding author upon reasonable request.

Received: 23 March 2018 Accepted: 12 July 2018

Published online: 06 August 2018

\section{References}

1. Cohen, S. B. et al. Protein composition of catalytically active human telomerase from immortal cells. Science 315, 1850-1853 (2007).

2. Kim, N. W. et al. Specific association of human telomerase activity with immortal cells and cancer. Science 266, 2011-2015 (1994).

3. Wong, M. S., Wright, W. E. \& Shay, J. W. Alternative splicing regulation of telomerase: a new paradigm? Trends Genet. 30, 430-438 (2014).

4. Kim, W. et al. Regulation of the human telomerase gene TERT by telomere position effect-over long distances (TPE-OLD): implications for aging and cancer. PLoS Biol. 14, e2000016 (2016).

5. Bell, R. J. et al. Understanding TERT promoter mutations: a common path to immortality. Mol. Cancer Res. 14, 315-323 (2016).

6. Braunschweig, U., Gueroussov, S., Plocik, A. M., Graveley, B. R. \& Blencowe, B. J. Dynamic integration of splicing within gene regulatory pathways. Cell 152, 1252-1269 (2013).

7. Hrdlickova, R., Nehyba, J. \& Bose, H. R. Jr. Alternatively spliced TERT variants lacking telomerase activity stimulate cell proliferation. Mol. Cell. Biol. 32, 4283-4296 (2012).

8. Kilian, A. et al. Isolation of a candidate human telomerase catalytic subunit gene, which reveals complex splicing patterns in different cell types. Hum. Mol. Genet. 6, 2011-2019 (1997).

9. Saeboe-Larssen, S., Fossberg, E. \& Gaudernack, G. Characterization of novel alternative splicing sites in human telomerase reverse transcriptase (hTERT) analysis of expression and mutual correlation in mRNA isoforms from normal and tumour tissues. BMC Mol. Biol. 7, 26 (2006).

10. Yi, X., Shay, J. W. \& Wright, W. E. Quantitation of telomerase components and hTERT mRNA splicing patterns in immortal human cells. Nucleic Acids Res. 29, 4818-4825 (2001).

11. Yi, X. et al. An alternate splicing variant of the human telomerase catalytic subunit inhibits telomerase activity. Neoplasia 2, 433-440 (2000).

12. Hrdlickova, R., Nehyba, J. \& Bose, H. R. Jr. Alternatively spliced telomerase reverse transcriptase variants lacking telomerase activity stimulate cell proliferation. Mol. Cell. Biol. 32, 4283-4296 (2012).

13. Wong, M. S. et al. Regulation of telomerase alternative splicing: a target for chemotherapy. Cell Rep. 3, 1028-1035 (2013).

14. Wong, M. S., Shay, J. W. \& Wright, W. E. Regulation of human telomerase splicing by RNA:RNA pairing. Nat. Commun. 5, 3306 (2014).

15. Listerman, I., Sun, J., Gazzaniga, F. S., Lukas, J. L. \& Blackburn, E. H. The major reverse transcriptase-incompetent splice variant of the human telomerase protein inhibits telomerase activity but protects from apoptosis. Cancer Res. 73, 2817-2828 (2013).

16. Barash, Y. et al. Deciphering the splicing code. Nature 465, 53-59 (2010).

17. Castello, A. et al. Insights into RNA biology from an atlas of mammalian mRNA-binding proteins. Cell 149, 1393-1406 (2012).

18. Gerstberger, S., Hafner, M., Ascano, M. \& Tuschl, T. Evolutionary conservation and expression of human RNA-binding proteins and their role in human genetic disease. Adv. Exp. Med. Biol. 825, 1-55 (2014).

19. Frink, R. E. et al. Telomerase inhibitor imetelstat has preclinical activity across the spectrum of non-small cell lung cancer oncogenotypes in a telomere length dependent manner. Oncotarget 7, 31639-31651 (2016).

20. Buckanovich, R. J., Posner, J. B. \& Darnell, R. B. Nova, the paraneoplastic Ri antigen, is homologous to an RNA-binding protein and is specifically expressed in the developing motor system. Neuron 11, 657-672 (1993).

21. Buckanovich, R. J., Yang, Y. Y. \& Darnell, R. B. The onconeural antigen Nova1 is a neuron-specific RNA-binding protein, the activity of which is inhibited by paraneoplastic antibodies. J. Neurosci. 16, 1114-1122 (1996).

22. Hsu, T. Y. et al. The spliceosome is a therapeutic vulnerability in MYC-driven cancer. Nature 525, 384-388 (2015).

23. Ludlow, A. T. et al. Quantitative telomerase enzyme activity determination using droplet digital PCR with single cell resolution. Nucleic Acids Res. 42, e104 (2014).

24. Schor, I. E., Gomez Acuna, L. I. \& Kornblihtt, A. R. Coupling between transcription and alternative splicing. Cancer Treat. Res. 158, 1-24 (2013).

25. Gimenez, M. et al. Quantitative proteomic analysis shows differentially expressed HSPB1 in glioblastoma as a discriminating short from long survival factor and NOVA1 as a differentiation factor between low-grade astrocytoma and oligodendroglioma. BMC Cancer 15, 481 (2015).

26. Knudsen, A. et al. Ri antibodies in patients with breast, ovarian or small cell lung cancer determined by a sensitive immunoprecipitation technique. Cancer Immunol. Immunother. 55, 1280-1284 (2006). 
27. Mancuso, M., Orsucci, D., Bacci, A., Caldarazzo Ienco, E. \& Siciliano, G. Anti$\mathrm{Ri}$-associated paraneoplastic cerebellar degeneration. Report of a case and revision of the literature. Arch. Ital. Biol. 149, 318-322 (2011).

28. Teplova, M. et al. Protein-RNA and protein-protein recognition by dual KH1/ 2 domains of the neuronal splicing factor Nova-1. Structure 19, 930-944 (2011).

29. Zhang, C. et al. Integrative modeling defines the Nova splicing-regulatory network and its combinatorial controls. Science 329, 439-443 (2010).

30. Gunes, C. \& Rudolph, K. L. The role of telomeres in stem cells and cancer. Cell 152, 390-393 (2013).

31. Kumar, M., Lechel, A. \& Gunes, C. Telomerase: the devil inside. Genes (Basel) 7, E43 (2016).

32. Villate, O. et al. Nova1 is a master regulator of alternative splicing in pancreatic beta cells. Nucleic Acids Res. 42, 11818-11830 (2014).

33. Darnell, R. B. RNA regulation in neurologic disease and cancer. Cancer Res. Treat. 42, 125-129 (2010).

34. Leggere, J. C. et al. NOVA regulates Dcc alternative splicing during neuronal migration and axon guidance in the spinal cord. eLife 5, e14264 (2016).

35. Saito, Y. et al. NOVA2-mediated RNA regulation is required for axonal pathfinding during development. eLife 5, e14371 (2016).

36. Eskiocak, U. et al. Functional parsing of driver mutations in the colorectal cancer genome reveals numerous suppressors of anchorage-independent growth. Cancer Res. 71, 4359-4365 (2011).

37. Ule, J. et al. An RNA map predicting Nova-dependent splicing regulation. Nature 444, 580-586 (2006).

38. Sei, E. \& Conrad, N. K. UV cross-linking of interacting RNA and protein in cultured cells. Methods Enzymol. 539, 53-66 (2014).

39. Dredge, B. K., Stefani, G., Engelhard, C. C. \& Darnell, R. B. Nova autoregulation reveals dual functions in neuronal splicing. EMBO J. 24, 1608-1620 (2005).

40. Jensen, K. B. et al. Nova-1 regulates neuron-specific alternative splicing and is essential for neuronal viability. Neuron 25, 359-371 (2000).

41. Bryan, T. M., Englezou, A., Dunham, M. A. \& Reddel, R. R. Telomere length dynamics in telomerase-positive immortal human cell populations. Exp. Cell Res. 239, 370-378 (1998)

42. Jafri, M. A., Ansari, S. A., Alqahtani, M. H. \& Shay, J. W. Roles of telomeres and telomerase in cancer, and advances in telomerase-targeted therapies. Genome Med. 8, 69 (2016).

43. Chen, L. Y. \& Lingner, J. AUF1/HnRNP D RNA binding protein functions in telomere maintenance. Mol. Cell 47, 1-2 (2012).

44. Pont, A. R., Sadri, N., Hsiao, S. J., Smith, S. \& Schneider, R. J. mRNA decay factor AUF1 maintains normal aging, telomere maintenance, and suppression of senescence by activation of telomerase transcription. Mol. Cell 47, 5-15 (2012).

45. Advani, V. M., Belew, A. T. \& Dinman, J. D. Yeast telomere maintenance is globally controlled by programmed ribosomal frameshifting and the nonsense-mediated mRNA decay pathway. Translation (Austin) 1, e24418 (2013).

46. Ding, J. et al. Crystal structure of the two-RRM domain of hnRNP A1 (UP1) complexed with single-stranded telomeric DNA. Genes Dev. 13, 1102-1115 (1999).

47. Redon, S., Zemp, I. \& Lingner, J. A three-state model for the regulation of telomerase by TERRA and hnRNPA1. Nucleic Acids Res. 41, 9117-9128 (2013).

48. Ford, L. P., Suh, J. M., Wright, W. E. \& Shay, J. W. Heterogeneous nuclear ribonucleoproteins $\mathrm{C} 1$ and $\mathrm{C} 2$ associate with the RNA component of human telomerase. Mol. Cell. Biol. 20, 9084-9091 (2000).

49. Jean-Philippe, J., Paz, S. \& Caputi, M. hnRNP A1: the Swiss army knife of gene expression. Int. J. Mol. Sci. 14, 18999-19024 (2013).

50. Le, P. N., Maranon, D. G., Altina, N. H., Battaglia, C. L. \& Bailey, S. M. TERRA, hnRNP A1, and DNA-PKcs interactions at human telomeres. Front. Oncol. 3, 91 (2013).

51. Zhang, Q. S., Manche, L., Xu, R. M. \& Krainer, A. R. hnRNP A1 associates with telomere ends and stimulates telomerase activity. RNA 12, 1116-1128 (2006).

52. Mallinjoud, P. et al. Endothelial, epithelial, and fibroblast cells exhibit specific splicing programs independently of their tissue of origin. Genome Res. 24, 511-521 (2014).

53. Salton, M. \& Misteli, T. Small molecule modulators of pre-mRNA splicing in cancer therapy. Trends Mol. Med. 22, 28-37 (2016).

54. Chung, J., Khadka, P. \& Chung, I. K. Nuclear import of hTERT requires a bipartite nuclear localization signal and Akt-mediated phosphorylation. J. Cell Sci. 125, 2684-2697 (2012).
55. Ryan, M. D., King, A. M. \& Thomas, G. P. Cleavage of foot-and-mouth disease virus polyprotein is mediated by residues located within a 19 amino acid sequence. J. Gen. Virol. 72(Pt 11), 2727-2732 (1991).

56. Ramirez, R. D. et al. Immortalization of human bronchial epithelial cells in the absence of viral oncoproteins. Cancer Res. 64, 9027-9034 (2004).

57. Zhang, L., Komurov, K., Wright, W. E. \& Shay, J. W. Identification of novel driver tumor suppressors through functional interrogation of putative passenger mutations in colorectal cancer. Int. J. Cancer 132, 732-737 (2013).

58. Ly, P. et al. RNAi screening of the human colorectal cancer genome identifies multifunctional tumor suppressors regulating epithelial cell invasion. Cell Res. 22, 1605-1608 (2012)

59. Ly, P. et al. Aneuploid human colonic epithelial cells are sensitive to AICARinduced growth inhibition through EGFR degradation. Oncogene 32, 3139-3146 (2013).

60. Zhao, Y. et al. Telomere extension occurs at most chromosome ends and is uncoupled from fill-in in human cancer cells. Cell 138, 463-475 (2009).

61. Lai, T. P., Wright, W. E. \& Shay, J. W. Generation of digoxigenin-incorporated probes to enhance DNA detection sensitivity. Biotechniques 60, 306-309 (2016).

\section{Acknowledgements}

We thank Drs. John Minna and Adi Gazdar for cell lines and transcriptomics. We acknowledge Henrietta Lacks and her family members for the important contributions "HeLa" cells have made to this work. This work was performed in laboratories constructed with support from NIH grant C06 RR30414. This work was supported by AG01228 from the National Institute on Aging (W.E.W. and J.W.S.) and by a T32 Training Grant CA124334 (J.W.S.). A Pathway to Independence award to ATL (K99/R00 CA197672-01A1, National Cancer Institute (NCI)). We also acknowledge the Harold Simmons NCI Designated Comprehensive Cancer Center Support Grant (CA142543), the NCI SPORE (CA070907), and the Southland Financial Corporation Distinguished Chair in Geriatric Research (J.W.S. and W.E.W.).

\section{Author contributions}

M.S.W. performed the minigene screen and analysis. A.T.L. conducted all other experiments. J.D.R., L.Y., and N.D. contributed to the RT-PCR and telomerase activity analysis. K.B. performed the bioinformatics. L.Z. helped perform the cancer growth assays and quantified the data. I.M. and L.Z. helped perform the xenograft studies. M.E.S and L.Y. performed the RNA bait assays and other assays. T.P.L. contributed reagents and assistance for telomere length analysis. A.T.L., M.S.W., J.W.S., and W.E.W. designed the study, analyzed the data, and wrote the manuscript. All authors discussed the results and commented/edited the manuscript.

\section{Additional information}

Supplementary Information accompanies this paper at https://doi.org/10.1038/s41467 018-05582-x.

Competing interests: The authors declare no competing interests.

Reprints and permission information is available online at http://npg.nature.com/ reprintsandpermissions/

Publisher's note: Springer Nature remains neutral with regard to jurisdictional claims in published maps and institutional affiliations.

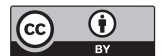

Open Access This article is licensed under a Creative Commons Attribution 4.0 International License, which permits use, sharing, adaptation, distribution and reproduction in any medium or format, as long as you give appropriate credit to the original author(s) and the source, provide a link to the Creative Commons license, and indicate if changes were made. The images or other third party material in this article are included in the article's Creative Commons license, unles indicated otherwise in a credit line to the material. If material is not included in the article's Creative Commons license and your intended use is not permitted by statutory regulation or exceeds the permitted use, you will need to obtain permission directly from the copyright holder. To view a copy of this license, visit http://creativecommons.org/ licenses/by/4.0/

(c) The Author(s) 2018 